\title{
Spectrum Handoff Management in Cognitive HetNet Systems Overlaid with Femtocells
}

\author{
Anwer Al-Dulaimi and Alagan Anpalagan \\ WINCORE Laboratory \\ Ryerson University, Toronto, Canada
}

\author{
John Cosmas \\ School of Engineering and Design \\ Brunel University, London, UK
}

\begin{abstract}
Cognitive radio networks can facilitate seamless mobility to users considering their effective use of the dynamic spectrum access. This is performed by proactive/reactive adaptation of transmission operations in response to the wireless environment changes. One of these operations includes handoff between various wireless domains. The handoff here is not just a registration with a new base station, but it is also a negotiation to get access to the available channels locally in coexistence with the primary users. This dynamic adaptation between channels known as spectrum handoff (SH) significantly impacts the time of handoff re-connection which raises many questions about the functioning of the cognitive radio solution in the next generation of network systems. Therefore, it is necessary to develop a new method for roaming mobile users especially networks that employ small-cells such as femtocells in order to reduce the unnecessary channel adaptations. This paper proposes a new entity namely channel assigning agent (CAA) for managing spectrum handoff, operator database, and channel access authentication. The goal of this mechanism is to retain the same channel used by a mobile user whenever possible to improve network performance by reducing the unnecessary spectrum handoffs. The modeling and efficiency of the proposed scheme are validated through simulation results. The proposed solution improves the accessibility of resources and stability of mobile radio connections that benefits mobile users as well as operators.
\end{abstract}

Index Terms - Cognitive radio, Femtocell, Mobility management, Spectrum handoff, Throughput

\section{INTRODUCTION}

$\mathrm{C}$ ognitive radio users may encounter multiple connections interruptions when channels becomes unavailable due to the primary users transmissions or normal handoff operations. These interruptions triggers in a series of Spectrum Handoffs (SHs) to restore cognitive communications whenever an interruption event occurs [1], [2]. Clearly, spectrum handoffs will increase the time required for re-connecting the newly arrival mobile users in any cognitive transmission domain while re-allocating channels. This new factor of time delay increases the time required for data delivery especially in cognitive networks that employ small cells such as femtocells. The main concern for such model of networks is that interruption may occur for unpredicted time intervals leading to significant signaling overhead and degradation in the whole system performance.

It reasonable to incorporate any new mobility functionality with the IP layer in order to solve the problem of spectrum handover. This is due to the fact that the IP layer is generic enough to serve all underlying technologies [3]. Also, it is widely accepted that there is a strong need to migrate the technology-specific core infrastructures toward all-IP networks [4]. The mobile IP working group within the internet engineering task force (IETF) proposed a packet-based mobility management protocol namely mobile internet protocol (MIP) in order to support global mobility in IP networks. This was upgraded later on to MIPv6 in response to the emergence of IPv6. In MIP, each mobile node (MN) is identified using two different IP addresses: a permanent home address (HoA) and a temporary care of address (CoA). The CoA is provided to the $\mathrm{MN}$ as it roams in a foreign network other than its home network. The CoA is given to the MN by the visiting subnet after issuing a router solicitation (RS) message to its foreign agent (FA) [5].

Even with the flexibility in performing IP handover, developing a solution for spectrum handoff in cognitive radio networks is still a complex challenge as it needs to answer those two questions: how to transfer the updated state information of the mobile users' used channel from the MIP to the Mobility Management Entity (MME)? what entities should be involved to allocate a certain channel for a mobile user that is moving between two cognitive access points? Considering the motivation to avoid the impacts of spectrum handover and the complexity of the above challenges, we propose a new scheme that can allocates the same channel to a cognitive mobile user moving between various access points as long as this channel is vacant in these domains. This goals that we intend to achieve here are:

- Reducing the number of unnecessary spectrum handoffs and improving the cognitive communications stability.

- Minimizing the time latency incurred during the data delivery.

- Improving spectrum coordination and avoiding channels scattering due to unnecessary spectrum handoff.

Therefore, the main contribution of this paper is to propose a solution to the spectrum handoff problem in cognitive radio networks overlaid with femtocells. We propose a new channel assigning agent (CAA) entity at the IP protocol layers. The CAA retain the same channel in use by a mobile unit when it movies to a new macro/femto transmission domain whenever 
the requested channel is available. The CAA is coupled with the MME to allow centralized management of the channel allocation during handoff in large cognitive networks. This can minimize or eliminate the time consumed during spectrum handoff in a cognitive radio network that employs femtocells. Our simulation results demonstrate that the proposed scheme reduces the total handoff latency, end-to-end data time delay, number of handoffs and improves the overall system throughput. The contents of this paper are arranged into stages in order to develop the CAA system model as shown in Fig. 1.



Fig. 1. Overview of contents in this paper.

The remainder of this paper is organized as follows. Section II discusses related studies. Section III overviews the IPv6 system model and the flow diagrams of the proposed scheme. Section IV describes the protocol of spectrum mobility and handoff in cognitive networks. The algorithms of spectrum handoff management are given in Section V. Simulation evaluations and performance analyses are presented in Section VI. Finally, our conclusions are presented in Section VII.

\section{RELATEd STUdies}

There are no literature that solve the problem of spectrum handoff using MIP according to the author's knowledge. Keeping in mind that there are few studies addressing solutions to the spectrum handoff issue, we will start by showing the most prominent solutions for spectrum handoff and then we show how MIP is used to solve the handoff latency. Our goal is to establish the necessary background for a solution that incorporates both SH and MIP as shown in the following sections. Some of the most related studies to our work are described below

An optimization problem was formulated in [6] for a proactive determination of target channel selection with the objective of minimizing the cumulative delay per connection.
This allows a newly arriving secondary user to avoid multiple spectrum handoffs due to the high-priority primary users and the traffic statistics of both the primary and the secondary users. This process takes into account the time required for channel switching and the transmission delay time resulting from accessing unsuitable channels. In contrast to a proactive assignment before accessing the channel, channel-switching (CW) policies and a proactive spectrum handoff protocol in [7] were proposed to let cognitive users vacate a channel before the primary user access it to avoid interference. This means that that cognitive user is using the channel and it acts before the primary user returns to occupy the space. Once the cognitive user driven into conduct a spectrum handoff, a distributed channel selection scheme is activated to avoid collisions among cognitive users in a multiuser spectrum handoff scenario. A further application of the optimal target channel sequence selection in proactivedecision spectrum handoff is given in [8] with a Poisson arriving of primary users. The theoretical analysis has shown a minimum probability of spectrum handoffs failure using the proposed scheme. The authors in [9] considered the channel handoff agility limitations for a cognitive radio user with a dynamic multichannel-access capability. The channel handoff agility was modeled as a continuous-time Markov process in order to analytically derive the forced access termination and blocking probabilities of cognitive users. Although the paper assumptions accepts that spectrum handoffs can only be performed to vacant channels that are immediate neighbors of the cognitive users' current channels but does not show how this scenario can reduce the numbers of spectrum handoffs or proactively prevent them. Clearly, the spectrum handoff studies investigates methods that can host the arrival of a cognitive user in the spectrum or the ways to response to subsequent changes in the spectrum availability without being able to prevent spectrum handoff occurrence.

The handling of handoff operations with the MIP have a very different prospective from the spectrum handoff prospective that we mentioned earlier due to the operation management. The MIP features allow to investigate the connection latency, state information of the mobile users and unifying technologies using IP layer. For example, the authors in [10] proposed an optimized handoff scheme using an adaptive retransmission timer that is proportional to the size of the messages involved in the transactions of the handoff process. This local mobility management which was placed in FMIPv6 and HMIPv6 have shown a major support to handle network layer mobility for VoIP traffic. This also allowed a minimum disruption for interAR movements, which are the most expected cases, and avoided triangular routing, which can harms VoIP services in mobile systems. The focus on IPv6 approach to provide solutions for mobility was also investigated in [11] where a generic framework for handoff techniques was combined with mobility management mechanisms at the IP layer in order to replace the well-performing soft handoff capabilities of UMTS/WLAN radio technologies. Although the IP handover mechanism was employed to manage the restoration of radio communication as well as proactively take actions and establish state information, the given analysis of the MIPv6 and Fast 
MIPv6 protocol operation focused only on handoff delays. A very similar solution using IPv6 can be also seen in [12] and [13] to support dynamic location changes of MNs and propose solutions for the impact of mobility. Analytical and simulation evaluations in [14], [15] and [16] addressed seamless mobility management by transferring the features of SIP of a separate location management function to the MIPv4. One of the advantages of such modelling is the support of a global seamless handover between homogeneous or heterogeneous networks (HetNets). However, mobility management becomes more challenging when migrating from macro-only to HetNet environments. This is due to the more challenging interference conditions, small cells appearing and disappearing more quickly as MN devices move, macro and small cells are deployed at different carrier frequencies, and so on [17]. All this literature on network-based mobility management protocols has not considered SH over IP layer.

In contrast to the aforementioned literature, this paper develops a long-term solution for the challenging spectrum handoff. Our solution aims at significantly reducing the number of spectrum handoffs in cognitive HetNets. This is performed by directly connecting the MIPv6 to the MME using a new entity that can stabilize the mobile user connections with fewer or no spectrum handoff events. The solution couples the correspondence node, target node and network management entity using IPv6 protocol as discussed in the next section.

\section{SOLUTION FRAMEWORK FOR SPECTRUM HANDOFF}

The HetNet have many small coverage cells such as femtocells than that of cellular systems. Hence, it is reasonable to predict that handoffs will be more frequent than that in only macro networks. In a cognitive radio network, this becomes more complicated with spectrum handoffs and the tremendous need for frequency adaptations when moving among various transmission domains. Therefore, any solution framework should enable to update in MIP networks with the channel used by a $\mathrm{MN}$ in order to maintain connection continuity of ongoing connections when the MN moves into a new domain. Our choice is to use MIPv6 for two reasons: Firstly, it enables a mobile node (MN) to keep the network connectivity even if the MN changes its point of attachment to the network [18] which is essential for HetNets with different technologies. Secondly, MIPv6 has a minimized control traffic [19] which is essential for an effective mobility management and dynamic wireless environment. The framework of the CAA solution suggests that the information on $\mathrm{MN}$ operative cognitive channel is exchanged among the MN, source AP, and the target AP for channel allocation. In the following subsections we show how the CAA integrated with the MIPv6 for HetNet spectrum handoff management. Then, we show the control signals for a mobility scheme that scans the channel availability and allocate channels between multiple domains. We also identify the conditions for channel assessment that can trigger spectrum handoff whenever necessary. These will formulate the basis for the CAA algorithms given in the following sections.

\section{A. Introducing the CAA}

In order to reduce or revoke the interruption time that occurs from frequent spectrum handoffs of a mobile user moving between different cognitive access points, we proposed in our earlier work in [20] to create a new agent namely channel assigning agent (CAA) based IPv4 for WLAN technologies. Although the functionality of proposed CAA was to allocate channels for cognitive users, it cannot provide roaming for a MN moving between different technologies/HetNet domains. In this paper, we develop further the CAA to allow LTE/WLAN systems to use the same agent to control spectrum handoff between variety of macro and femto domains. In this extended new application, the CAA is incorporated within the MIPv6 to support roaming option as well as spectrum handoff control signaling. The current known mobility management in cognitive radio network does not support channel allocation; therefore, it is necessary to incorporate such functionality in order to be able to deal with the spectrum handoff problem. As CAA is integrated to the IPv6 protocol, IP-dynamic host configuration protocol (DHCP) is used to create the global interface for all cognitive radio clients in motion. This simplifies the process of registration and allow a central management for channel allocation as proposed by the CAA. To perform a seamless handoff, we allow mobile user to communicate directly with its correspondent nodes $(\mathrm{CN})$ instead of tunneling the traffic via home agent (HA) node especially inside femtocells. This utilizes local transmission opportunities efficiently in small cells communications as one of our main goals in this paper. Additionally, a two-way handshake (Solicit/Reply) is used instead of the usual four-way handshake (Solicit/Advertise, Request/Reply) to reduce the time of response while adapting performing handoff and/or spectrum handoff operations. This is a very essential requirement for mobile users moving at high speeds.

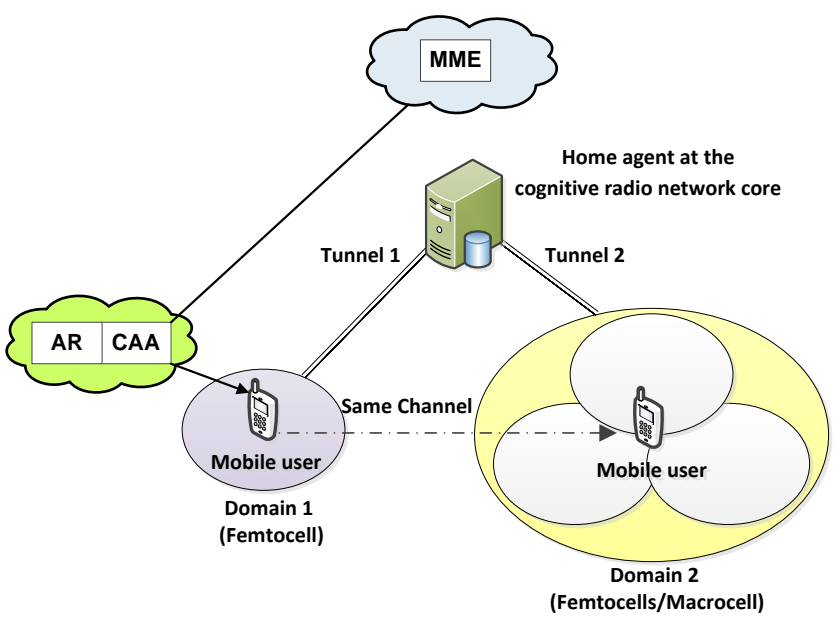

Fig. 2. The CAA entity as part of IP protocol

As mentioned earlier, the framework solution incorporates the CAA at the IP network layer to assign certain channels prior to any handoff actions. This assumes that the CAA is aware of the channel used by the $\mathrm{MN}$ and it can determines the 
availability of the same channel at the target subcell domain prior to any new handoff request. This means that the CAA is also aware of the mobile user route of movement. As such information is very hard to be predicated, we assume that the CAA in real applications can learns from long-term monitoring of MNs. For example, the CAA can predict the route of a certain mobile user who is used to take the same highway street to commute to work at early morning and at the end of the working day. Such long-term data of monitored users and the locations registrations obtained from the access router (AR) allow the CAA to allocate channels in collaboration with the MME, as shown in Fig. 2. Hence, the CAA operation procedures include: Firstly, informing the target node to assign a certain channel $\left(f_{i}\right)$ at the time of arrival of a MN in order to interface communications immediately and eliminate any need to perform spectrum handoff. Secondly, the CAA determines the obtained channel sensing reports to manage the allocation of another channel $\left(f_{i}^{\prime}\right)$ and adopt this new channel as the new operative channel for this $\mathrm{MN}$ to avoid further spectrum handoff actions. The last scenario is very likely to occur in cognitive HetNets but the CAA functionality keeps the handoff mostly as a horizontal-handoff over a series of connections rather than a vertical-handoff where a MN adapts rapidly between various channels.

Considering Fig. 2, when a MN moves from domain 1 to domain 2, the tunnel 1 is terminated at the time of MN registration with the access point at domain 2. Using the CAA, we should be able to revoke the impacts of interruption time that is likely to occur due to the spectrum handoff operation. This advantage of CAA application do not eliminate the other usual handoff time latencies that occurs due to the normal mobility registration and signaling operations. Although this solution is designed to be a general application to all size cognitive radio network domains, this study will focus on femtocell-to-macrocell network scenario model. This limitation allow us to develop the spectrum handoff signal control flow for a network management of a macrocell of LTE technology overlaid with femtocells of WLAN technologies. This spectrum handoff solution is further developed into a channel assignment protocol that can retain mobile user channel during handoff between different wireless domains. These contributions are proposed in the following sections of this paper.

\section{B. Model Formulation}

We investigate the spectrum handoff problem that is likely to occur for a MN travelling between two cognitive base stations. Our intension is to develop the necessary control signal scheme for channel allocation for HetNet. From the literature, the authors in [21] proposed an intercell spectrum handoff scheme as shown in Fig. 3. In this scheme, the mobile user senses the spectrum periodically to detect the presence of any primary user. The sensing results are exchanged with $\mathrm{CN}$ which may decides to allocate another channel to the $\mathrm{MN}$. In this case, the availability of the new channel will be negotiated with neighbor nodes to prevent interference. If it was decided to adapt to a new channel, there will be a distribution in the services during the time of frequency adaptation. This disrupted mobile user needs to carry out additional intercell handoff to maintain a connection. In the worst case, the mobile users must carry out a new network entry procedure due to the connection loss. Clearly, this was developed to solve the problem when a primary user reclaims its channel and the cognitive $\mathrm{MN}$ has to look for another channel to maintain cognitive communications. We think that this scheme is an intial step to generate the signal control scheme for the spectrum handoff and we expand this work by incorporating a channel reservation mechanism that can reduce not only the probability of service interruption, but also the total number of potential handoffs.

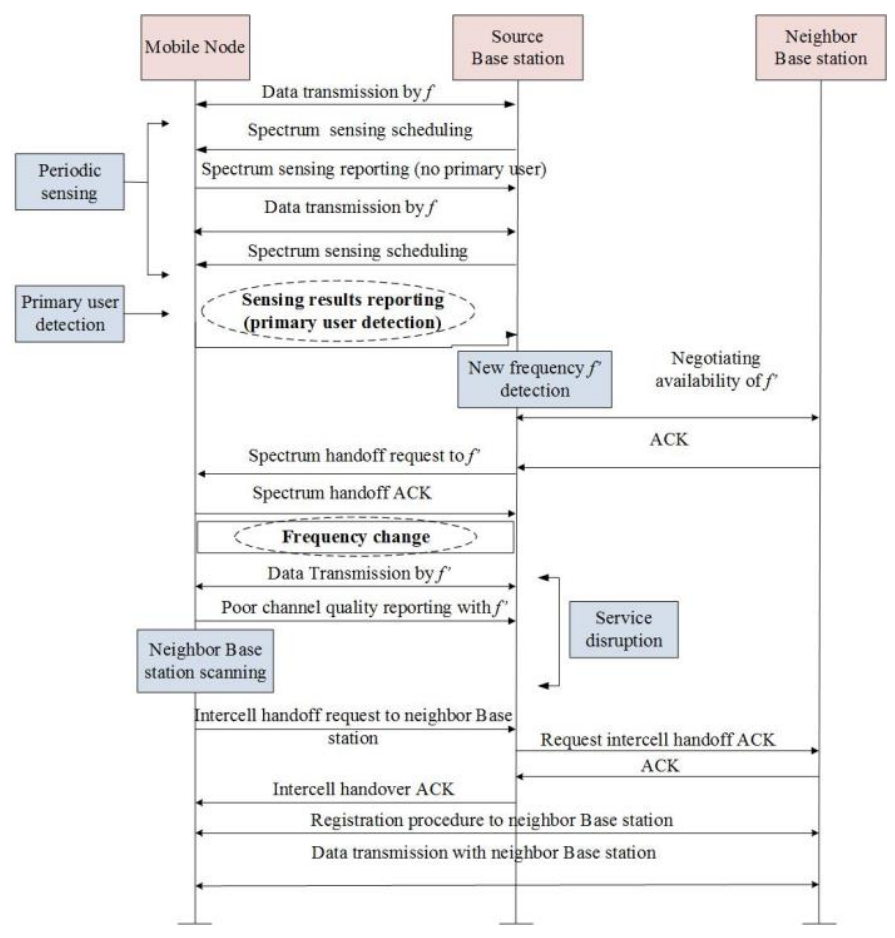

Fig. 3. Operation of intercell spectrum handoff [21].



Fig. 4. Inter/intra cell spectrum handoffs.

Considering Fig. 4, there are two potential cases for spectrum handoffs: inter-spectrum handoff within the cell, and intraspectrum handoff within the subcell. In fact, all of them can be treated as the same using one spectrum handoff procedure in a 
spectrum pool model. Thus, the channel reservation concept can be generalized to all users subject to the channels availability and accessibility at any of the wireless domains. Specifically, the goal is to reduce/eliminate unnecessary spectrum handoffs for active radio connections. Although such channel reservation model can impact the way of assigning the spectrum between cognitive users, we will justify this proposal in the following sections by showing the improvement in performance of active connections. Therefore, we generalize the conditions to enforce or cease a spectrum handoff operations to all mobile nodes moving between any two domains as shown in Fig. 3 as following:

Condition 1: Enforcing spectrum handoff:

$$
\left\{Q\left(f_{i}^{\prime}\right)<\delta_{t h 1}\right\} \cap\left\{l \text {, s.t. } Q\left(\ddot{f_{l}}\right)>\delta_{t h 2}\right\}
$$

Condition 2: Ceasing spectrum handoff:

$$
\left\{Q\left(f_{i}\right)<\delta_{t h 1}\right\} \cap\left\{Q\left(f_{i}^{\prime}\right)>\delta_{t h 2}\right\}
$$

where $f_{i}$ is the original frequency of serving base station, $f_{i}^{\prime}$ is the new frequency of the target base station after spectrum handoff, $\ddot{f}_{l}$ is the frequency of the neighbor base station indexed

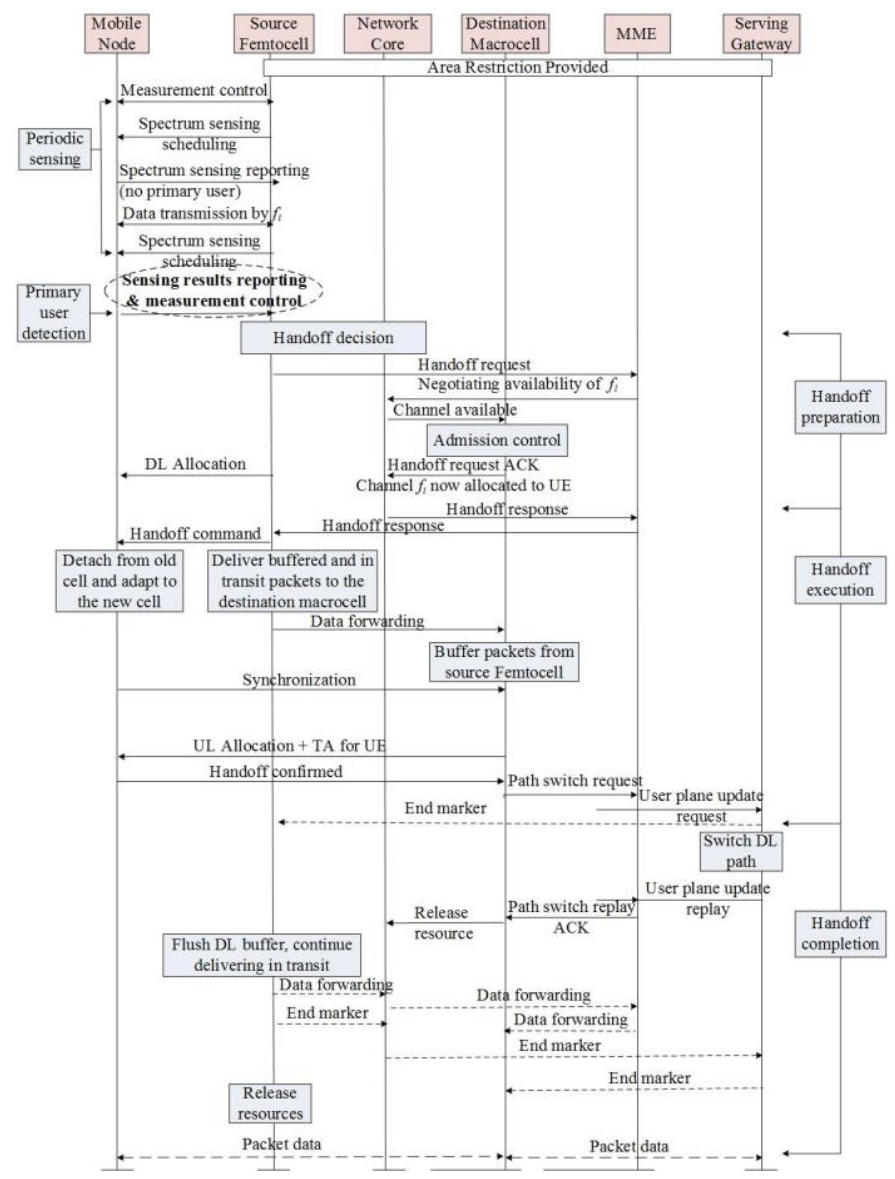

Fig. 5. Scheme for spectrum handoff while changing to $\left(f_{i}^{\prime}\right)$. by $l, \delta_{t h 1}$ is the threshold for triggering spectrum handoff, $\delta_{t h 2}$ is the threshold for determining spectrum handoff, while $Q$ is defined as the received signal to noise ratio (SNR) for each frequency channel.

Since the handoff model given in Fig. 3 is not able to meet the requirements of large-size networks due to the absence of a central management unit that allocates channels between various domains, we provide a new spectrum handoff scheme that is extendable to cognitive HetNets that employ small cells of femtocells. The new signal flow diagrams for channels allocation are given the following subsection.

\section{The New Handoff Scheme}

The spectrum handoff scheme for a mobile user travelling from femto-to-macro domains is given in Fig. 5. The scheme determines the availability of the frequency $\left(f_{i}\right)$ for the new arrival mobile user in order to maintain the same channel in the new target domain.

If the enforcing condition of spectrum handoff given in (1) is satisfied, the detailed procedure shown in Fig. 6 is carried out. When a cognitive user is moving towards macrocell domain, a control message is reported with the latest updates of the periodic spectrum sensing. Then, a handoff request is made to the next base station provided by the frequency of operation $\left(f_{i}\right)$ to negotiate the availability of this channel at the new domain.

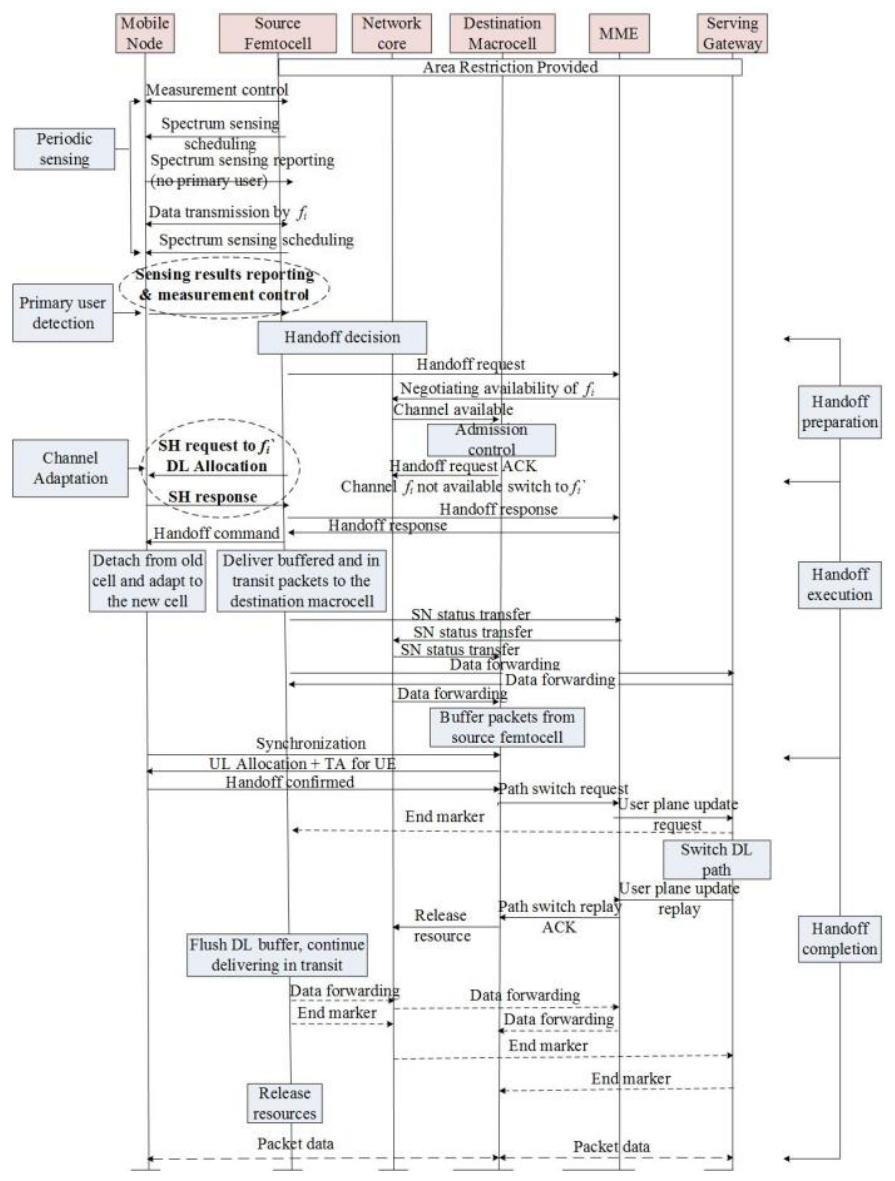

Fig. 6. Scheme for spectrum handoff using $\left(f_{i}\right)$. 
If the channel is not available at the target base station, a channel scanning is performed to identify a new available channel $\left(f_{i}^{\prime}\right)$. Once $\left(f_{i}^{\prime}\right)$ becomes available, a channel adaption request is sent back to the mobile node to change to $\left(f_{i}^{\prime}\right)$. Before adapting to a new frequency, the MN buffers the data and halts any transmission. Similarly, the femtocell buffers and directs any packets to the macrocell unit in order to resume normal transmissions. Thus, a path switch request is issued to the cognitive radio network core which acts as the serving gateway to redirect future communications to the new route of connections as well as updating the user profile the new location and frequency.

Fig. 6 shows the spectrum handoff scheme in case of frequency $\left(f_{i}\right)$ is available at the macrocell for the newly arrived mobile node. Comparing Fig. 5 and Fig. 6, we can see clearly the differences in the control signals and the additional operations required for the frequency adaptation scenario. This confirms the impact of spectrum handoff that adds an extra delay time to the reconnection time incurred during normal handoff action. There are other time delays incurred in cognitive systems due to the spectrum handoff such as: time required for scanning the spectrum, channel assignment, and frequency reconfiguration.

In the next section, we provide the mechanism for the CAA functionality to verify the given solution of holding or adapting channels between various cognitive base stations.

\section{SPECTRUM HANDOFF MECHANISM}

The framework for spectrum handoff solution proposed in this paper assumes that the mobile node sends its location data, used channel and sensing data message during handoff request. Upon receiving, the MME starts making predictions of the channel availability before the current transmission frame ends. Based on these predictions, the MME decides whether to allocate the same channel to the mobile node or to switch to a new channel or terminate the ongoing transmission. In this section, we develop a new assessment model that can determine the channel availability and use these data to help the MME making decisions on spectrum handoff requests. We propose two criterion for channel assessment (a) the forecast probability that the current candidate channel (i.e., a channel that can be selected for continuing the current data transmission) is busy or idle and (b) the expected length of the channel idle period. Based on these measures, we design spectrum handoff policies that are used to assign channels between various cognitive users.

To estimate the probability that a channel is idle, it is necessary to identify the time intervals of busy and idle states of random transmission durations. Considering Fig. 7 and using a Bayesian learning algorithm [22], the probability that channel $i$ is idle can be given as follows:

$$
P_{f_{i}}^{i d l e}(t)=\frac{X_{i}(t)+1}{X_{b}(t)+X_{i}(t)+2}
$$

where $X_{b}(t)$ and $X_{i}(t)$ are the numbers of slot times that channel $i$ is busy or idle in a future time interval $t$.

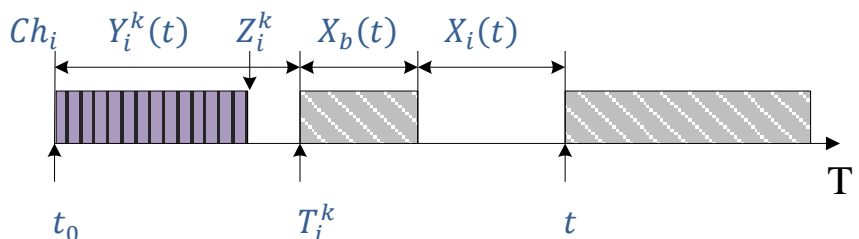

Fig. 7. Primary user traffic activity on channel $f_{i}$,

TABLE I

MYTHOLOGY

$Y_{i}^{k}(t)$ Number of successfully transmitted packets of a number of cognitive user $k$ over channel $i$

$Y_{f}^{k}(t)$ Number of failed transmitted packets of a number of cognitive user $k$ over channel $i$

$T_{i}^{k} \quad$ Packets arrival time of a number of cognitive user $k$ over channel $i$

$X_{b}(t)$ Number of slot times that channel $i$ is busy in a future time interval $t$

$X_{i}(t)$ Number of slot times that channel $i$ is idle in a future time interval $t$

$k^{\text {th }} \quad$ Number of cognitive users

$i^{\text {th }} \quad$ Number of channels available to cognitive users

$Z_{i}^{k} \quad$ Packet length of a cognitive user $k$ over channel $i$

Therefore, the criterion for channel $i^{\text {th }}$ to become a candidate channel at time interval $t$ is given by:

$$
\left\{\begin{array}{c}
P_{f_{i}}^{\text {success }}(t)=\frac{Y_{i}^{k}(t)+1}{Y_{i}^{k}(t)+Y_{f}^{k}(t)+2} \text { and } T_{i}^{k} \leq t \\
Z_{i}^{k} \leq \tau_{z}
\end{array}\right.
$$

where $Y_{i}^{k}(t)$ and $Y_{f}^{k}(t)$ are the number of successful and failed cognitive radio slot transmissions over channel $i, Z_{i}^{k}$ represents the packet length of the a number $k^{\text {th }}$ of cognitive users on channel $i$ and $\tau_{z}$ is the maximum packet length that can be conveyed over a link ( $\tau_{z}=10 \mathrm{~ms}$ for SIP).

Therefore, the probability of successful cognitive transmission over certain link using (4) can be shown in Fig. 8.

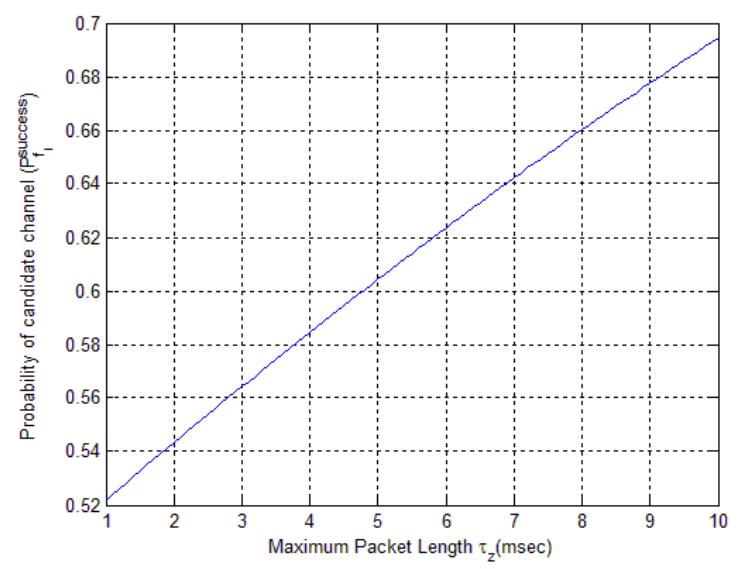

Fig. 8. Probability of successful candidate channel for cognitive transmission over interval time $t$

In this case, there is no need to perform a spectrum handoff operation and the cognitive user is being facilitated easily in coexistence with the primary user. The condition in (4) means that, in order to support at least one cognitive user frame, the 
probability that the duration of the idleness of the $i^{\text {th }}$ channel has to be longer than a frame size must be higher than or equal to $\tau_{z}$.

This means that a spectrum handoff action will be necessary whenever:

$\left(P_{f_{i}}^{\text {success }}(t)=0\right)$ if $T_{i}^{k}<t$ and $T_{i}^{k}+\mathrm{Z}_{i}^{k} \geq \mathrm{t}, \quad k \geq 1$,

$$
\begin{gathered}
\left(P_{f_{i}}^{\text {success }}(t)=0\right) \text { if } T_{i}^{k}+\mathrm{Z}_{i}^{k}<t \text { and } T_{i}^{k+1} \geq \mathrm{t}, k \geq 1, \\
T_{i}^{k+1} \geq \mathrm{t}, \quad k=0,
\end{gathered}
$$

In this case it is necessary to perform spectrum handoff provided by scanning other channels in the available band to identify a new candidate channel in order to resume transmission. It should be also notice that a cognitive user should switch to a new channel if:

$$
Z_{i}^{k} \geq \tau_{z}
$$

The above polices are used to develop the probe for channel assignment in spectrum handoff model. This performed in the CAA protocol given in the following section.

\section{Protocol Modelling}

\section{A. Channel Availability Algorithm}

We propose a protocol that conducts a spectrum handoff using channel assessment model that was given in the previous section. The protocol has two parts. The first part, namely Algorithm 1 (the pseudo-code highlights the channel allocation scheme in Fig. 5), describes how a cognitive user initiates a handoff request. Regardless of the transmission domain, if a handoff request arrives at the base station, the MME use the channel availability predications to allocate a certain channel as reported by CAA. Based on the prediction results, if the channel satisfies the policies in (4) for data transmissions, the MME sends ACK that frequency channel $f_{i}$ is available to the MN and the transmission resumes at the beginning of the next time frame. Upon allocating the channel the mobile node detached from the prior base station, performs the normal handoff and starts the data transmission using the same channel.

\begin{tabular}{lc}
\hline$/{ }^{* *}$ Algorithm 1: Starting A Handoff Request: $f_{i}$ is available ${ }^{* *} / /$ \\
\hline $\mathbf{1}$ & Mobile node arrives at new base station domain \\
2 & Handoff request: CAA reports used channel, $C h_{i}=f_{i}, H O=0$ \\
3 & Scanning for $\left(f_{i}\right):$ frequency and time duration (4) \\
$\mathbf{4}$ & \{ \\
$\mathbf{5}$ & If $\left(f_{i}\right)$ is available \\
$\mathbf{6}$ & $M M E$ allocates $f_{i}$ at the new destination (2)
\end{tabular}

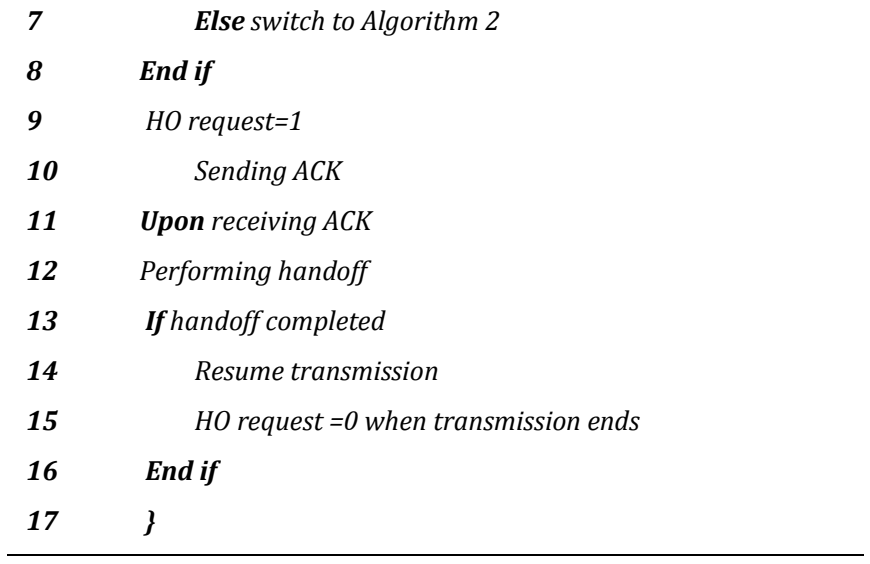

\section{B. Channel Adaptation Algorithm}

The second part, namely Algorithm 2 (the pseudo-code highlights the algorithm in Fig. 6), is a spectrum handoff when channel $f_{i}$ is not available for MN transmission at the target base station. This protocol determines the process for $\mathrm{MN}$ to carry out a spectrum handoff as in (1) and then switch to a new channel by the time the current frame in transmission ends. This should happen when the channel sensing information satisfies the policies in (5) and (6) for a potential spectrum handoff. If the condition is not fulfilled, then the used channel will be available for the next frame transmission and we will switch to use Algorithm (1). Once the condition is fulfilled and a spectrum handoff is necessary, the MME evaluates the set of channels available for cognitive transmission.

The algorithm maintains two functions of (NUC) and (LSC) as the number and the list of the candidate channels for cognitive transmissions, respectively, similar to [7]. The MME evaluates the next candidate channel on the LSC using the (4). Then, the MME sends a channel-adaptation-request (CAR) packet containing the updated chosen channel information in the next time slot. Upon receiving the CAR packet, the cognitive mobile node replies with an acknowledgement (ACK) packet. As the ACK packet is successfully received by the source base station, the mobile node performs a spectrum handoff by the end of the frame to avoid any interference to the primary user. A connection is established between the mobile node and the target base station while data communications are re-routed to the next linked base station.

\begin{tabular}{lc}
\hline$/^{* * * * *}$ Algorithm $2:$ Spectrum Handoff: $f_{i}$ is not available ${ }^{* * * * *} / /$ \\
\hline $\mathbf{1}$ & Initialize operation \\
$\mathbf{2}$ & $C A R=0, H O=0, N U C=0, L S C=0$ \\
$\mathbf{4}$ & For $(i=0, i \leq M)$ do \\
$\mathbf{5}$ & Predicting the availability of Ch (channels other than $\left.\left(f_{i}\right)\right):$ \\
6 & End for \\
7 & If $\left(C h_{i}, 0 \leq i \leq M\right)$ available: frequency and time duration $(4)$ \\
8 & $N U C=N U C+1$ \\
9 & $L S C(N U C)=i$
\end{tabular}




$\begin{array}{ll}10 & \text { End if } \\ 11 & \text { If } L S C=0 \\ 12 & \text { Go to step } 4 \\ 13 & \text { End if } \\ 14 & \text { Upon receiving CAR then } \\ & \text { Send ACK } \\ 15 & \text { Performing complete handoff (including SH) } \\ 16 & \text { Start scanning the channel } \\ 17 & \text { If channel get busy } \\ 18 & \text { Transmission stops } \\ 19 & \text { Go to step } 4 \\ 20 & \text { Else HO request }=1 \\ 21 & \text { End if } \\ 22 & \text { If HO request }=1 \\ 23 & \text { Transmitting data } \\ 24 & \text { HO request }=0 \text { when transmission ends } \\ 25 & \text { End if } \\ 26 & \text { ? } \\ 27 & \text { Go to Algorithm } 1\end{array}$

The time delay of the spectrum handoff is defined as the interval from the time a cognitive user leaves its used channel to the time it resumes the transmission on a new channel. There is also a possibility that the allocation is not appropriate as the primary user resumes transmission over the new channel. Therefore, it is necessary for the cognitive mobile user to scan the channel and make sure that it is idle at the beginning of any frame transmission. If the channel is sensed busy, the Algorithm 2 is launched again to search for another channel.

\section{Vi. Performance Evaluation}

\section{A. Network Setup}

The developed spectrum handoff scheme is compared with conventional handoff management model using the designed OPNET models. The choice for using this software is due to the fact that examining the performance of higher level layers such as TCP/IP is complex challenge that needs to be solved with a very powerful computing processing system. The OPNET is capable of simulating complex heterogeneous networks of multiple numbers of nodes provided by the capability to mimic real time networks operations [23]. The channel allocation algorithms are coded and incorporated within the functions of the simulator. The simulations integrate cognitive radio network models with primary network to create dynamic channel selectivity similar to what a cognitive network experiences in real operations. The network parameters for the designed simulations are shown in Table II.

The proposed algorithms are set up to have no frequency channel overlap. Therefore, transmissions from an instance of one model can only be received by instances of the same model. Hence, instead of trying all receiver channels every time, we
TABLE II

SIMULATION PARAMETER VALUES

\begin{tabular}{|c|c|}
\hline Parameter & Value \\
\hline Cell layout & $\begin{array}{l}\text { Sectors: } 1 \text { macrocell, } 3 \\
\text { femtocells, and } 14 \text { primary units }\end{array}$ \\
\hline Users active per sector & 2 \\
\hline Minimum distance to BS & 35 meters \\
\hline Propagation model & Hata-large city \\
\hline Number of available channels $i^{t h}$ & 14 \\
\hline Packet inter-arrival time $Y_{i}^{K}$ & $10 \mathrm{~ms}$ \\
\hline Voice packet length $Z_{i}^{k}$ & 80 bytes \\
\hline$t_{\text {OFF }}$ & variable \\
\hline \multicolumn{2}{|l|}{ Application Layer } \\
\hline Encoder Scheme & G.711 (silence) \\
\hline Voice Frames Per Packet & 1 \\
\hline Type of Service & Best Effort $(0)$ \\
\hline Signaling & SIP \\
\hline Max. ACK Delay & $0.2 \mathrm{sec}$ \\
\hline Max. ACK Segments & 2 \\
\hline Fast Recovery & Reno \\
\hline \multicolumn{2}{|l|}{ Cognitive Network } \\
\hline Physical characteristics & OFDM $(802.11 \mathrm{a})$ \\
\hline Data rate & $48 \mathrm{Mbps}$ \\
\hline maximum transmission power & $1 \mathrm{~mW}$ \\
\hline Route request rate limit & $10 \mathrm{pkts} / \mathrm{sec}$ \\
\hline Node Traversal Time & $0.04 \mathrm{sec}$ \\
\hline \multicolumn{2}{|l|}{ Primary Network } \\
\hline Physical characteristics & Direct sequence \\
\hline Data rate & $11 \mathrm{Mbps}$ \\
\hline Channel bandwidth & $22 \mathrm{MHz}$ \\
\hline Max. Receive lifetime & $0.5 \mathrm{secs}$ \\
\hline
\end{tabular}

filtered these out by the prior information on users' channels. There are two primary places to do that filtering: the receiver group pipeline stage and the channel match pipeline stage. In the receiver group, the code access, channel minimum frequency, and bandwidth attributes for the transmitter/receiver channels uses the information on channels to accept or reject the pair between base station and mobile node. The default receiver group pipeline stage does not pay attention to frequency or bandwidth attributes.

We use the attribute dra_chanmatch which is a compiled pipeline procedure to dynamically compute the type of interaction which can occur between a radio transmitter channel and a radio receiver channel. The default model can dictate that a transmission be viewed by a radio receiver channel as a valid and potentially receivable signal. This allow us to avoid any interference or an irrelevant signal. If the latter outcome occurs, the remainder of the pipeline is not executed for the given transmitter-receiver pair. 


\section{B. System Level Simulation}

Spectrum handoff is another source besides traditional handoff for providing mobility in cognitive radio network architecture. Considering spectrum handoff, this dynamic process of adaptation between channels may occur to fixed and moving nodes at the same time. However, splitting the effects of conventional handoff schemes from the new spectrum handoff is a new topic for discussion in secondary networks. Our goal is to reduce/eliminate the time of interruption in services that can occur during the adaptation of a cognitive mobile node between different channels. The evaluation setup of the proposed spectrum handoff method is performed by allowing the mobile node shown in Fig. 9 to use the same channel when moving between positions \#1-to-\#2, \#3, \#4, \#5 and then back to \#1. This was compared to the conventional model where mobile node maybe forced to adapt to another frequency channel when performing normal handoff between two wireless domains.

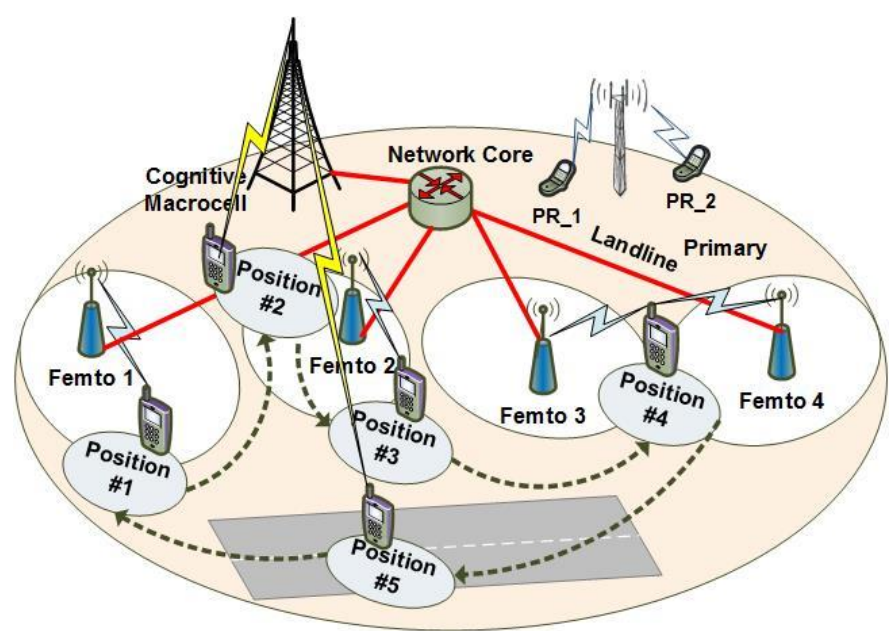

Fig. 9. Spectrum handoffs events for a cognitive mobile station moves between different femto and macro domains.

Considering the system in Fig. 9, the mobile node experiences five different events of handoff as it moves along the trajectory shown in the figure. These handoffs occur when:

1. The mobile node moves out of the femtocell coverage area (position\#1) where service capacity is much higher because of the stable and short range communications towards macrocell coverage zone.

2. The mobile node arrives the femtocell coverage area (position\#2) leaving the macrocell services area.

3. The mobile mode heads directly to the macrocell area (position\#3) leaving the femtocell zone of node 2.

4. The mobile node moves to the fully covered area by three transmission sources: the macrocell and two femtocells (position\#4).

5. The mobile node moves along the motorway (position\#5) returning to its initial point (position\#1).

To examine the performance of the new spectrum handoff scheme using the scenario above, the mobile node is set to use different speeds of movement along the route shown in the figure. These speeds were set according to Table III which presents the vehicular speed limits.

TABLE III

SIMULATED MOBILE USER SPEEDS

\begin{tabular}{|c|c|}
\hline Speed (mph) & Description \\
\hline 2.877 & Pedestrian \\
\hline 20 & Cars speed in urban areas \\
\hline 30 & Cars speed in urban areas \& villages \\
\hline 40 & Cars speed in non-built areas \\
\hline 50 & Cars speed in non-built areas \\
\hline 70 & National speed limit \\
\hline
\end{tabular}

An IP telephony and silence suppressed signals are generated to test the system performance. The reason to choose this kind of application is that normal phone calls are actually composed of different times of activity where the user is either talking or silent. The IP networks transmit packets only when the data and control information are in action. Therefore, there is no usage for the channel if the clients are not sending anything. Thus, such application is very useful in analyzing the cognitive networks and the dynamic spectrum access models. The reason for this is that transmissions occur temporarily and when it is needed only which is the same principles of the cognitive radio systems.

The simulation setup includes two networks, primary and secondary networks that coexist with each other. Primary users are transmitting using all channels while secondary systems are accessing the available band on temporary basis whenever there are no primary activities. In order to simulate the performance of the new model precisely, the number of mobile users is set to 1,3 , and 7 respectively. In each case study, an evaluation for the system improvement with no spectrum handoff is compared to the traditional case [11], [16], [18], where the spectrum handoff is happening along the movement route.

\section{Results}

In this section, the simulation results are presented to validate the proposed scheme.

In Fig. 10, handoff time latency is shown for all the simulated numbers of mobile nodes as a function of the mobile speed. The figure depicts that there is a considerable time savings using the new scheme of CAA compared to the conventional model of handoff. The figure shows that the savings in handoff time latency increase as the number of mobile nodes increases. This signifies the importance and success of the proposed solution in practical applications that employ large numbers of cell and subcell domains. This reduction in time latency during handoff shows that a mobile user can quickly be re-connected to the destination base station and services can be maintained without interruptions. 


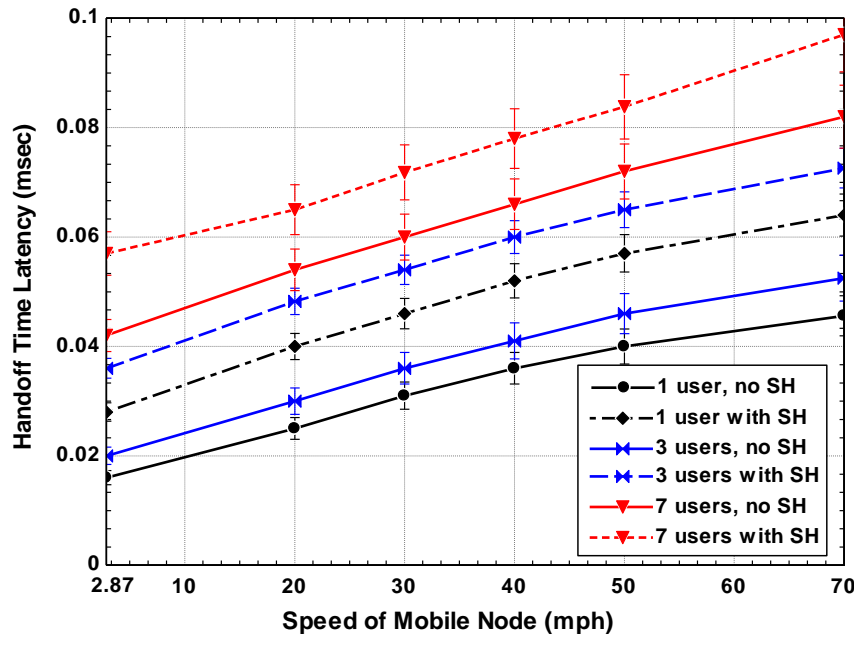

Fig. 10. Handoff time latency as a function for the mobile speed.



Fig. 11. End-to end time delays as a function for the mobile speed.

Fig. 11 compares the end-to-end time delays for the different numbers of users who with and without are experiencing the spectrum handoff $(\mathrm{SH})$ events. In all of the above cases, the end-to-end delay time is increasing with higher mobile user speeds. The reason for this is that major time delays are incurred as the speeds of cognitive mobile nodes increase causing more handoffs to occur as users move between various femto and macro domains. However, the cancellation of spectrum handoff that results from the installation of CAA entity provides in much lower time delays as depicted by the figure for all simulated cases.

The throughput in Fig. 12 shows also a major improvement with the application of CAA and no SH events. For all simulated cases, the throughput is higher than the case for the traditional spectrum handoff. The explanation for this is that the time spent in the adaptation between various channels reduces the performance of the system. This interruption time impacts the overall time delay in Fig. 11 and the throughput in Fig. 12.
It can be noticed also that the performance of the simulated system is declining slowly with the increment of the mobile nodes speeds. This is due to the fact that a speedy mobile node loses some local transmission opportunities that are available at scattered locations in the macro and femto domains. Therefore, the faster mobile nodes become the lowest ability to attain local transmission opportunities.

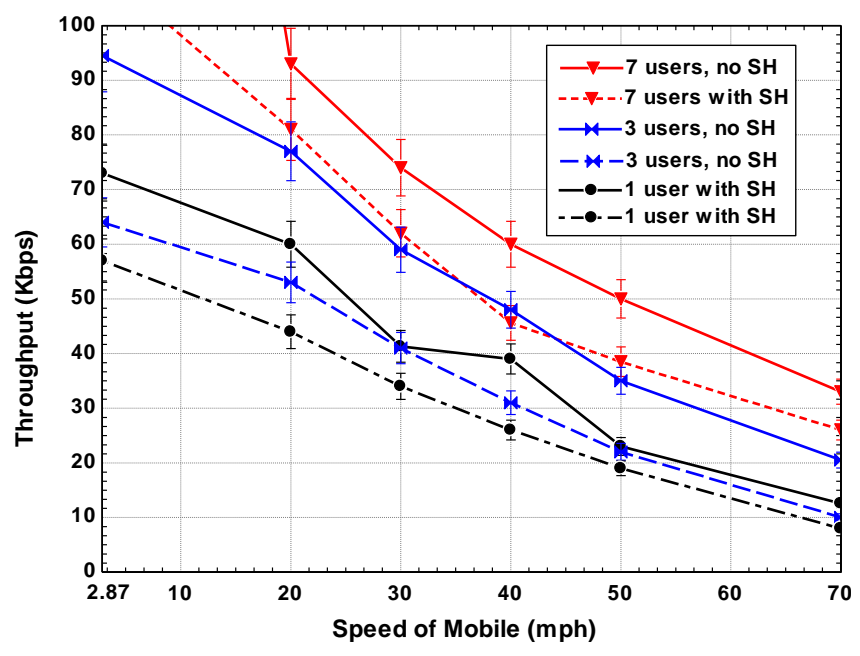

Fig. 12. Throughput as a function for the mobile speed.

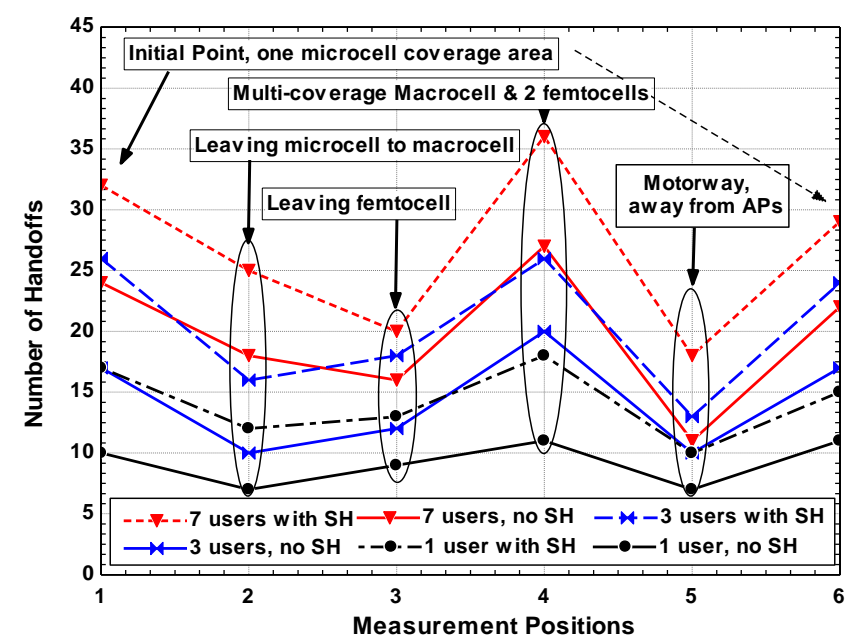

Fig. 13. Number of handoffs vs. mobile locations as shown in Fig. 9.

Figure 13 shows the reduction in the number of handoffs due to the CAA installation. Clearly, there is lower number of handoffs occurring when mobile nodes travel between the different coverage areas as shown in Fig. 8. The measurement points were selected to show the improvement in performance at the most prominent points of spectrum handoff events. It can be noticed that the number of handoffs is increasing as the mobile nodes move towards the macrocell base station (position\#1-to-\#2). The maximum value can be seen when mobile nodes are within the coverage area of three sources: macrocell and two femtocells at position\#4. Afterwards, 
handoff shows the lowest values as the mobile nodes travel along the motorway within macrocell coverage at position\#5. At the end, the handoff values increases again as the mobile nodes return home to the initial point of their journey (position\#1). Generally, more handoffs occur while moving between heterogeneous domains rather than homogenous domains or one domain scenario.

In summary, the CAA entity that maintains channels to cognitive mobile nodes, improves the performance in accessing the spectrum and reduces the numbers of handoffs incurred due to the frequency adaptations.

\section{CONCLUSION}

A new scheme is proposed to reduce the spectrum handoff in future cognitive radio networks that employ small cells such as femtocells. A new entity named as channel assigning agent is introduced at the mobile IP to allocate the same channel used by a cognitive mobile user as it moves between sub-cell areas. The main goal of this design is to reduce the interruption time that occurs during frequency adaptation and the redundant unnecessary spectrum handoffs for a mobile user travelling at various speeds. The solution involved the design of handoff algorithms that scans the available band for the channel in operation before any decision of adapting to other frequencies. Then, a comprehensive assessment was conducted to evaluate the suitability of the free time interval within the selected channel to host the cognitive mobile node packets. Results show considerable improvement in throughput with less number of handoffs and major savings in time delay using the proposed scheme.

\section{REFERENCES}

[1] C.-W. Wang and L.-C. Wang, "Analysis of Reactive Spectrum Handoff in Cognitive Radio Networks," Selected Areas in Communications, IEEE Journal on, vol. 30, no. 10, pp. 2016 2028, November 2012.

[2] Y.-S. Chen and J.-S. Hong, "A Relay-Assisted Protocol for Spectrum Mobility and Handover in Cognitive LTE Networks," Systems Journal, IEEE, vol. 7, no. 1, pp. 77 - 91, March 2013.

[3] Z. Yan and J.-H. Lee, "State-Aware Pointer Forwarding Scheme With Fast Handover Support in a PMIPv6 Domain," Systems Journal, IEEE, vol. 7, no. 1, pp. 92 - 101, March 2013.

[4] C. Makaya and S. Pierre, "An Architecture for Seamless Mobility Support in IP-Based Next-Generation Wireless Networks," Vehicular Technology, IEEE Transactions on, vol. 57, no. 2, pp. 1209 - 1225, March 2008.

[5] T. Taleb, and K. Letaief, "A cooperative diversity based handoff management scheme," Wireless Communications, IEEE Transactions on, vol. 9, no. 4, pp. 1462 - 1471, April 2010.

[6] L.-C. Wang, C.-W. Wang, and C.-J. Chang, "Optimal Target Channel Sequence Design for Multiple Spectrum Handoffs in Cognitive Radio Networks," Communications, IEEE Transactions on, vol. 60, no. 9, pp. 2444 - 2455, September 2012.

[7] Y. Song and J. Xie, "ProSpect: A Proactive Spectrum Handoff Framework for Cognitive Radio Ad Hoc Networks without Common Control Channel," Mobile Computing, IEEE Transactions on, vol. 11, no. 7, pp. 1127 - 1139, July 2012.

[8] S. Zheng, X. Yang, S. Chen, and C. Lou, "Target Channel Sequence Selection Scheme for Proactive-Decision Spectrum
Handoff," Communications Letters, IEEE, vol. 15, no. 12, pp. 1332 - 1334, December 2011.

[9] M. NoroozOliaee, B. Hamdaoui, X. Cheng, T. Znati, and M. Guizani, "Analyzing Cognitive Network Access Efficiency Under Limited Spectrum Handoff Agility," Vehicular Technology, IEEE Transactions on, vol. 63, no. 3, pp. 1402 1407, March 2014.

[10] H. Fathi, S.S. Chakraborty, R. Prasad, "Optimization of Mobile IPv6-Based Handovers to Support VoIP Services in Wireless Heterogeneous Networks," Vehicular Technology, IEEE Transactions on, vol. 56, no. 1, pp. 260 - 270, Jan. 2007.

[11] L. Dimopoulou, G. Leoleis, and IS. Venieris, "Fast handover support in a WLAN environment: challenges and perspectives," Network, IEEE, vol. 19, no. 3, pp. 14 - 20, MayJune 2005.

[12] M.-C. Chuang, J.-F. Lee, and M.-C. Chen, "SPAM: A Secure Password Authentication Mechanism for Seamless Handover in Proxy Mobile IPv6 Networks," IEEE Systems Journal, vol. 7, no. 1, pp. 102 - 113, March 2013.

[13] S. Cespedes, L. Ning S. Xuemin, "VIP-WAVE: On the Feasibility of IP Communications in 802.11p Vehicular Networks," IEEE Transactions on Intelligent Transportation Systems, vol. 14, no. 1, pp. 82 - 97, March 2013.

[14] H. Ali-Ahmad, X. Lagrange, M. Ouzzif, and P. Bertin, "Comparative Cost Analysis on Global Mobility Management Schemes in IPv6 Mobile Networks," Vehicular Technology Conference (VTC Spring), 2013 IEEE 77th, pp. 1 - 6, 2-5 June 2013.

[15] C.-M. Huang, C.-H. Lee, and J.-R. Zheng, “A Novel SIP-Based Route Optimization for Network Mobility," Selected Areas in Communications, IEEE Journal on, vol. 24, no. 9, pp. 1682 1691, Sept. 2006.

[16] J.-T. Park, S.-M. Chun, J.-H. Choi, and S.-M. Lee, "Simple mobility management protocol for global seamless handover," Consumer Communications and Networking Conference (CCNC), 2012 IEEE, pp. 677 - 681, 14-17 Jan. 2012.

[17] K.I Pedersen, P.H. Michaelsen, C. Rosa, and S. Barbera, "Mobility enhancements for LTE-advanced multilayer networks with inter-site carrier aggregation," Communications Magazine, IEEE, vol. 51, no. 5, pp. 64 - 71, May 2013.

[18] T. Kim and J. Lee, "Mobility and QoS support in 4G wireless networks," Communications and Networks, Journal of, vol. 7, no. 2, pp. 178 - 191, June 2005.

[19] T. Taleb, A. Jamalipour, Y. Nemoto, and N. Kato, "DEMAPS: A Load-Transition-Based Mobility Management Scheme for an Efficient Selection of MAP in Mobile IPv6 Networks," Vehicular Technology, IEEE Transactions on, vol. 58, no. 2, pp. 954 - 965, Feb. 2009.

[20] A. Al-Dulaimi, S. Al-Rubaye, and J. Cosmas, "Adaptive Coexistence between Cognitive and Fibre Networks," in Wireless Multi-Access Environments and Quality of Service Provisioning: Solutions and Application. G. M. Muntean and R. Trestian, Ed. Pennsylvania, USA: IGI Global, pp. 311 - 336, 2011.

[21] O. Jo and D.-H. Cho, "Seamless spectrum handover considering differential path-loss in cognitive radio systems," Communications Letters, IEEE, vol. 13, no. 3, pp. 190 - 192, March 2009.

[22] A. Motamedi and A. Bahai, "MAC Protocol Design for Spectrum-agile Wireless Networks: Stochastic Control Approach," New Frontiers in Dynamic Spectrum Access Networks, 2007. DySPAN 2007. 2nd IEEE International Symposium on, pp. 448 - 451, 17-20 April 2007.

[23] A. Al-Dulaimi, H.S. Al-Raweshidy, J. Cosmas, and J. Loo, "Cognitive Mesh Networks," Vehicular Technology Magazine, IEEE, vol. 5, no. 3, pp. 54 - 60, Sept. 2010. 


\section{Response Letter}

Dear editors:

Firstly, we would like to thank you for the time and efforts that you spent on this paper as well as all the reviewers. Your comments have really helped us to significantly improve the paper contents and the presentation of our contributions. We have addressed all comments by you and all reviewers one by one in this revised version that we are submitting to your kind consideration. We wish that we did successfully responded to all comments.

Compared with the previous version, the main changes of this version are as follows.

1. We carefully addressed all comments. Please see the following summary for our response to the reviewers' comments.

2. We added introduction, related work, and mathematical evaluation in this new version.

3. We further polish the writing of the paper again and again.

4. We marked (in blue color) the main changes in our paper. 


\section{Summary of Changes}

\section{Manuscript ID: ISJ-RE-14-02886}

\section{Reviewer 1:}

Comments 1: Related study and introduction are not appropriate.

Response: Thanks for your comments. We are sorry that we did not present enough information and differentiation from prior art. In the revised paper, we added related work and rearrange the introduction section. Considering the limited space in the original manuscript, we explain our main idea and contributions in detail as follows.

\section{INTRODUCTION}

ognitive radio users may encounter multiple connections interruptions when channels becomes

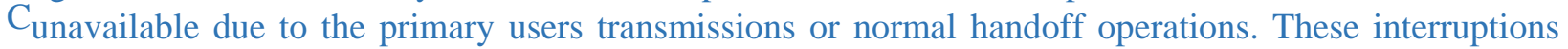
triggers in a series of Spectrum Handoffs (SHs) to restore cognitive communications whenever an interruption event occurs [1], [2]. Clearly, spectrum handoffs will increase the time required for reconnecting the newly arrival mobile users in any cognitive transmission domain while re-allocating channels. This new factor of time delay increases the time required for data delivery especially in cognitive networks that employ small cells such as femtocells. The main concern for such model of networks is that interruption may occur for unpredicted time intervals leading to significant signaling overhead and degradation in the whole system performance.

It reasonable to incorporate any new mobility functionality with the IP layer in order to solve the problem of spectrum handover. This is due to the fact that the IP layer is generic enough to serve all underlying technologies [3]. Also, it is widely accepted that there is a strong need to migrate the technology-specific core infrastructures toward all-IP networks [4]. The mobile IP working group within the internet engineering task force (IETF) proposed a packet-based mobility management protocol namely mobile internet protocol (MIP) in order to support global mobility in IP networks. This was upgraded later on to MIPv6 in response to the emergence of IPv6. In MIP, each mobile node (MN) is identified using two different IP addresses: a permanent home address (HoA) and a temporary care of address (CoA). The CoA is provided to the $\mathrm{MN}$ as it roams in a foreign network other than its home network. The CoA is given to the $\mathrm{MN}$ by the visiting subnet after issuing a router solicitation (RS) message to its foreign agent (FA) [5].

Even with the flexibility in performing IP handover, developing a solution for spectrum handoff in cognitive radio networks is still a complex challenge as it needs to answer those two questions: how to transfer the updated state information of the mobile users' used channel from the MIP to the Mobility Management Entity (MME)? what entities should be involved to allocate a certain channel for a mobile user that is moving between two cognitive access points? Considering the motivation to avoid the impacts of spectrum handover and the complexity of the above challenges, we propose a new scheme that can allocates the same channel to a cognitive mobile user moving between various access points as long as this channel is vacant in these domains. This goals that we intend to achieve here are: 
- Reducing the number of unnecessary spectrum handoffs and improving the cognitive communications stability.

- Minimizing the time latency incurred during the data delivery.

- Improving spectrum coordination and avoiding channels scattering due to unnecessary spectrum handoff.

Therefore, the main contribution of this paper is to propose a solution to the spectrum handoff problem in cognitive radio networks overlaid with femtocells. We propose a new channel assigning agent (CAA) entity at the IP protocol layers. The CAA retain the same channel in use by a mobile unit when it movies to a new macro/femto transmission domain whenever the requested channel is available. The CAA is coupled with the MME to allow centralized management of the channel allocation during handoff in large cognitive networks. This can minimize or eliminate the time consumed during spectrum handoff in a cognitive radio network that employs femtocells. Our simulation results demonstrate that the proposed scheme reduces the total handoff latency, end-to-end data time delay, number of handoffs and improves the overall system throughput. The contents of this paper are arranged into stages in order to develop the CAA system model as shown in Fig. 1.

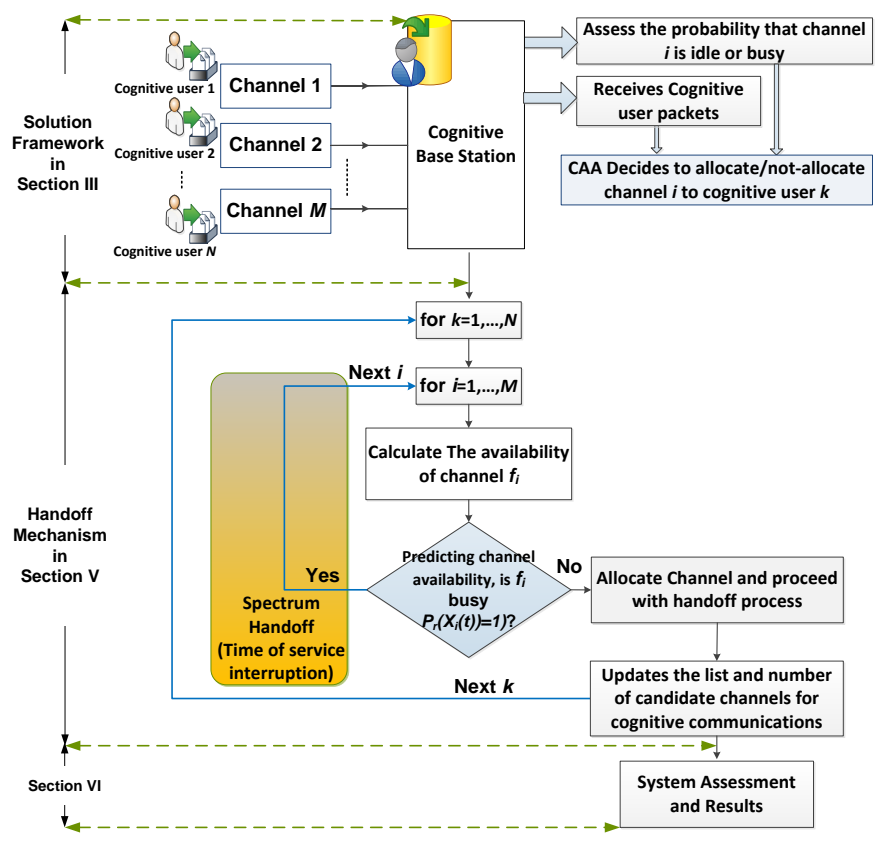

Fig. 1. Overview of contents in this paper.

The remainder of this paper is organized as follows. Section II discusses related studies. Section III overviews the IPv6 system model and the flow diagrams of the proposed scheme. Section IV describes the protocol of spectrum mobility and handoff in cognitive networks. The algorithms of spectrum handoff management are given in Section V. Simulation evaluations and performance analyses are presented in Section VI. Finally, our conclusions are presented in Section VII.

\section{RELATED STUDIES}

There are no literature that solve the problem of spectrum handoff using MIP according to the author's knowledge. Keeping in mind that there are few studies addressing solutions to the spectrum handoff issue, 
we will start by showing the most prominent solutions for spectrum handoff and then we show how MIP is used to solve the handoff latency. Our goal is to establish the necessary background for a solution that incorporates both SH and MIP as shown in the following sections. Some of the most related studies to our work are described below

An optimization problem was formulated in [6] for a proactive determination of target channel selection with the objective of minimizing the cumulative delay per connection. This allows a newly arriving secondary user to avoid multiple spectrum handoffs due to the high-priority primary users and the traffic statistics of both the primary and the secondary users. This process takes into account the time required for channel switching and the transmission delay time resulting from accessing unsuitable channels. In contrast to a proactive assignment before accessing the channel, channel-switching $(\mathrm{CW})$ policies and a proactive spectrum handoff protocol in [7] were proposed to let cognitive users vacate a channel before the primary user access it to avoid interference. This means that that cognitive user is using the channel and it acts before the primary user returns to occupy the space. Once the cognitive user driven into conduct a spectrum handoff, a distributed channel selection scheme is activated to avoid collisions among cognitive users in a multiuser spectrum handoff scenario. A further application of the optimal target channel sequence selection in proactive-decision spectrum handoff is given in [8] with a Poisson arriving of primary users. The theoretical analysis has shown a minimum probability of spectrum handoffs failure using the proposed scheme. The authors in [9] considered the channel handoff agility limitations for a cognitive radio user with a dynamic multichannel-access capability. The channel handoff agility was modeled as a continuous-time Markov process in order to analytically derive the forced access termination and blocking probabilities of cognitive users. Although the paper assumptions accepts that spectrum handoffs can only be performed to vacant channels that are immediate neighbors of the cognitive users' current channels but does not show how this scenario can reduce the numbers of spectrum handoffs or proactively prevent them. Clearly, the spectrum handoff studies investigates methods that can host the arrival of a cognitive user in the spectrum or the ways to response to subsequent changes in the spectrum availability without being able to prevent spectrum handoff occurrence.

The handling of handoff operations with the MIP have a very different prospective from the spectrum handoff prospective that we mentioned earlier due to the operation management. The MIP features allow to investigate the connection latency, state information of the mobile users and unifying technologies using IP layer. For example, the authors in [10] proposed an optimized handoff scheme using an adaptive retransmission timer that is proportional to the size of the messages involved in the transactions of the handoff process. This local mobility management which was placed in FMIPv6 and HMIPv6 have shown a major support to handle network layer mobility for VoIP traffic. This also allowed a minimum disruption for inter-AR movements, which are the most expected cases, and avoided triangular routing, which can harms VoIP services in mobile systems. The focus on IPv6 approach to provide solutions for mobility was also investigated in [11] where a generic framework for handoff techniques was combined with mobility management mechanisms at the IP layer in order to replace the well-performing soft handoff capabilities of UMTS/WLAN radio technologies. Although the IP handover mechanism was employed to manage the restoration of radio communication as well as proactively take actions and establish state information, the given analysis of the MIPv6 and Fast MIPv6 protocol operation focused only on handoff delays. A very similar solution using IPv6 can be also seen in [12] and [13] to support dynamic location changes of MNs and propose solutions for the impact of mobility. Analytical and simulation evaluations in [14], [15] and [16] addressed seamless mobility management by transferring the features of SIP of a separate location management function to the MIPv4. One of the advantages of such modelling is the support of a global seamless handover between homogeneous or heterogeneous networks (HetNets). However, mobility management becomes more challenging when migrating from macro-only to HetNet environments. This is due to the more challenging interference conditions, small cells appearing and disappearing more quickly as MN devices move, macro and small cells are deployed at different carrier frequencies, and so on [17]. 
All this literature on network-based mobility management protocols has not considered SH over IP layer.

In contrast to the aforementioned literature, this paper develops a long-term solution for the challenging spectrum handoff. Our solution aims at significantly reducing the number of spectrum handoffs in cognitive HetNets. This is performed by directly connecting the MIPv6 to the MME using a new entity that can stabilize the mobile user connections with fewer or no spectrum handoff events. The solution couples the correspondence node, target node and network management entity using IPv6 protocol as discussed in the next section.

\section{REFERENCES}

[1] C.-W. Wang and L.-C. Wang, "Analysis of Reactive Spectrum Handoff in Cognitive Radio Networks," Selected Areas in Communications, IEEE Journal on, vol. 30, no. 10, pp. 2016 - 2028, November 2012.

[2] Y.-S. Chen and J.-S. Hong, "A Relay-Assisted Protocol for Spectrum Mobility and Handover in Cognitive LTE Networks," Systems Journal, IEEE, vol. 7, no. 1, pp. 77 - 91, March 2013.

[3] Z. Yan and J.-H. Lee, "State-Aware Pointer Forwarding Scheme With Fast Handover Support in a PMIPv6 Domain," Systems Journal, IEEE, vol. 7, no. 1, pp. 92 - 101, March 2013.

[4] C. Makaya and S. Pierre, "An Architecture for Seamless Mobility Support in IP-Based Next-Generation Wireless Networks," Vehicular Technology, IEEE Transactions on, vol. 57, no. 2, pp. 1209 - 1225, March 2008.

[5] T. Taleb, and K. Letaief, "A cooperative diversity based handoff management scheme," Wireless Communications, IEEE Transactions on, vol. 9, no. 4, pp. 1462 - 1471, April 2010.

[6] L.-C. Wang, C.-W. Wang, and C.-J. Chang, "Optimal Target Channel Sequence Design for Multiple Spectrum Handoffs in Cognitive Radio Networks," Communications, IEEE Transactions on, vol. 60, no. 9, pp. 2444 - 2455, September 2012.

[7] Y. Song and J. Xie, "ProSpect: A Proactive Spectrum Handoff Framework for Cognitive Radio Ad Hoc Networks without Common Control Channel," Mobile Computing, IEEE Transactions on, vol. 11, no. 7, pp. 1127 - 1139, July 2012.

[8] S. Zheng, X. Yang, S. Chen, and C. Lou, "Target Channel Sequence Selection Scheme for ProactiveDecision Spectrum Handoff," Communications Letters, IEEE, vol. 15, no. 12, pp. 1332 - 1334, December 2011.

[9] M. NoroozOliaee, B. Hamdaoui, X. Cheng, T. Znati, and M. Guizani, "Analyzing Cognitive Network Access Efficiency Under Limited Spectrum Handoff Agility," Vehicular Technology, IEEE Transactions on, vol. 63, no. 3, pp. 1402 - 1407, March 2014.

[10]H. Fathi, S.S. Chakraborty, R. Prasad, "Optimization of Mobile IPv6-Based Handovers to Support VoIP Services in Wireless Heterogeneous Networks," Vehicular Technology, IEEE Transactions on, vol. 56, no. 1, pp. 260 - 270, Jan. 2007.

[11]L. Dimopoulou, G. Leoleis, and IS. Venieris, "Fast handover support in a WLAN environment: challenges and perspectives," Network, IEEE, vol. 19, no. 3, pp. 14 - 20, May-June 2005.

[12]M.-C. Chuang, J.-F. Lee, and M.-C. Chen, "SPAM: A Secure Password Authentication Mechanism for Seamless Handover in Proxy Mobile IPv6 Networks," IEEE Systems Journal, vol. 7, no. 1, pp. 102 - 113, March 2013.

[13]S. Cespedes, L. Ning S. Xuemin, "VIP-WAVE: On the Feasibility of IP Communications in 802.11p Vehicular Networks," IEEE Transactions on Intelligent Transportation Systems, vol. 14, no. 1, pp. 82 97, March 2013.

[14]H. Ali-Ahmad, X. Lagrange, M. Ouzzif, and P. Bertin, "Comparative Cost Analysis on Global Mobility Management Schemes in IPv6 Mobile Networks," Vehicular Technology Conference (VTC Spring), 2013 IEEE 77th, pp. 1 - 6, 2-5 June 2013. 
[15]C.-M. Huang, C.-H. Lee, and J.-R. Zheng, "A Novel SIP-Based Route Optimization for Network Mobility," Selected Areas in Communications, IEEE Journal on, vol. 24, no. 9, pp. 1682 - 1691, Sept. 2006.

[16]J.-T. Park, S.-M. Chun, J.-H. Choi, and S.-M. Lee, "Simple mobility management protocol for global seamless handover," Consumer Communications and Networking Conference (CCNC), 2012 IEEE, pp. 677 - 681, 14-17 Jan. 2012.

[17]K.I Pedersen, P.H. Michaelsen, C. Rosa, and S. Barbera, "Mobility enhancements for LTE-advanced multilayer networks with inter-site carrier aggregation," Communications Magazine, IEEE, vol. 51, no. 5, pp. 64 - 71, May 2013.

Comment 2: Inclusion of ipv6 with cognitive environment is not very convincing, need elaborations.

Response: Thanks for your comments. Since the handoff and mobility is highly related to the IPv6, we add description about handoff management and operations to Sections I \& II. We rearrange Section III, and illustrate the choice of incorporating the new proposed CAA entity within the IPv6 in the manuscript. We wish that this revision will help readers to understand the motivation behind using IPv6.

\section{SOLUTION FRAMEWORK FOR SPECTRUM HANDOFF}

The HetNet have many small coverage cells such as femtocells than that of cellular systems. Hence, it is reasonable to predict that handoffs will be more frequent than that in only macro networks. In a cognitive radio network, this becomes more complicated with spectrum handoffs and the tremendous need for frequency adaptations when moving among various transmission domains. Therefore, any solution framework should enable to update in MIP networks with the channel used by a MN in order to maintain connection continuity of ongoing connections when the MN moves into a new domain. Our choice is to use MIPv6 for two reasons: Firstly, it enables a mobile node (MN) to keep the network connectivity even if the MN changes its point of attachment to the network [18] which is essential for HetNets with different technologies. Secondly, MIPv6 has a minimized control traffic [19] which is essential for an effective mobility management and dynamic wireless environment. The framework of the CAA solution suggests that the information on MN operative cognitive channel is exchanged among the MN, source AP, and the target AP for channel allocation. In the following subsections we show how the CAA integrated with the MIPv6 for HetNet spectrum handoff management. Then, we show the control signals for a mobility scheme that scans the channel availability and allocate channels between multiple domains. We also identify the conditions for channel assessment that can trigger spectrum handoff whenever necessary. These will formulate the basis for the CAA algorithms given in the following sections.

\section{A. Introducing the $C A A$}

In order to reduce or revoke the interruption time that occurs from frequent spectrum handoffs of a mobile user moving between different cognitive access points, we proposed in our earlier work in [20] to 
create a new agent namely channel assigning agent (CAA) based IPv4 for WLAN technologies. Although the functionality of proposed CAA was to allocate channels for cognitive users, it cannot provide roaming for a MN moving between different technologies/HetNet domains. In this paper, we develop further the CAA to allow LTE/WLAN systems to use the same agent to control spectrum handoff between variety of macro and femto domains. In this extended new application, the CAA is incorporated within the MIPv6 to support roaming option as well as spectrum handoff control signaling.

[18]T. Kim and J. Lee, "Mobility and QoS support in 4G wireless networks," Communications and Networks, Journal of, vol. 7, no. 2, pp. 178 - 191, June 2005.

[19] T. Taleb, A. Jamalipour, Y. Nemoto, and N. Kato, "DEMAPS: A Load-Transition-Based Mobility Management Scheme for an Efficient Selection of MAP in Mobile IPv6 Networks," Vehicular Technology, IEEE Transactions on, vol. 58, no. 2, pp. 954 - 965, Feb. 2009.

[20]A. Al-Dulaimi, S. Al-Rubaye, and J. Cosmas, "Adaptive Coexistence between Cognitive and Fibre Networks," in Wireless Multi-Access Environments and Quality of Service Provisioning: Solutions and Application. G. M. Muntean and R. Trestian, Ed. Pennsylvania, USA: IGI Global, pp. 311 - 336, 2011.

Comment 3: spectrum hand-off mechanism may be elaborated in more detail.

Response: Thanks for your comments. In this new version, we provide more comprehensive details on spectrum handoff problem challenges, current solutions, and our proposed solution throughout this version to enable readers to understand the basics, problem and solutions of this problem. These can be seen in Sections I, III, III, and IV.

Comment 4: Simulation seems to be self-compared, with "SH" and "without SH" it is recommended to compare your results with some reputed existing work.

Response: Thanks for your comments. We are really sorry for not presenting enough information in the paper about this case. In this revised version we make it clear to the readers that <with "SH"> is meant to describe our proposed cognitive radio networks that can avoid spectrum handoff actions during handoff operations between different wireless domains. Such amendment to handoffs can happen only with the new proposed CAA entity as clarified in the paper. While $<$ without "SH"> describes the traditional cognitive radio networks where handoff operations incorporates spectrum handoff actions when a mobile node performs cell/intercell handoffs. As 
developed our algorithms using OPNET software, we were able to create the traditional cognitive radio systems with $\mathrm{SH}$ and then we developed the new nodes with the CAA to enable the channel reservation model that can maintain the same channel and avoid SH. Both models were simulated, at one time to show the advantages of the new development under the same channel availability profiles and traffic loads. This was described in Section VI in details.

Comment 5: Improve presentation and readability of time line diagrams.

Response: Thanks for your comments. We have worked carefully to improve the presentation and readability of time line diagrams in this revised version using new tools that increase the resolution and appearance of figures. 


\title{
Reviewer 2:
}

Comments 1: The motivation is clear, while the review on related work should be refined in section 2 .

\begin{abstract}
Response: Thanks for your comments. We are sorry that we did not present enough information and differentiation from prior art. In the revised paper, we added related work and shown how our work will be different from the literature. Considering the limited space in the original manuscript, we explain our main idea and contributions in detail as follows.
\end{abstract}

\section{Related Studies}

There are no literature that solve the problem of spectrum handoff using MIP according to the author's knowledge. Keeping in mind that there are few studies addressing solutions to the spectrum handoff issue, we will start by showing the most prominent solutions for spectrum handoff and then we show how MIP is used to solve the handoff latency. Our goal is to establish the necessary background for a solution that incorporates both SH and MIP as shown in the following sections. Some of the most related studies to our work are described below

An optimization problem was formulated in [6] for a proactive determination of target channel selection with the objective of minimizing the cumulative delay per connection. This allows a newly arriving secondary user to avoid multiple spectrum handoffs due to the high-priority primary users and the traffic statistics of both the primary and the secondary users. This process takes into account the time required for channel switching and the transmission delay time resulting from accessing unsuitable channels. In contrast to a proactive assignment before accessing the channel, channel-switching $(\mathrm{CW})$ policies and a proactive spectrum handoff protocol in [7] were proposed to let cognitive users vacate a channel before the primary user access it to avoid interference. This means that that cognitive user is using the channel and it acts before the primary user returns to occupy the space. Once the cognitive user driven into conduct a spectrum handoff, a distributed channel selection scheme is activated to avoid collisions among cognitive users in a multiuser spectrum handoff scenario. A further application of the optimal target channel sequence selection in proactive-decision spectrum handoff is given in [8] with a Poisson arriving of primary users. The theoretical analysis has shown a minimum probability of spectrum handoffs failure using the proposed scheme. The authors in [9] considered the channel handoff agility limitations for a cognitive radio user with a dynamic multichannel-access capability. The channel handoff agility was modeled as a continuous-time Markov process in order to analytically derive the forced access termination and blocking probabilities of cognitive users. Although the paper assumptions accepts that spectrum handoffs can only be performed to vacant channels that are immediate neighbors of the cognitive users' current channels but does not show how this scenario can reduce the numbers of spectrum handoffs or proactively prevent them. Clearly, the spectrum handoff studies investigates methods that can host the arrival of a cognitive user in the spectrum or the ways to response to subsequent changes in the spectrum availability without being able to prevent spectrum handoff occurrence.

The handling of handoff operations with the MIP have a very different prospective from the spectrum handoff prospective that we mentioned earlier due to the operation management. The MIP features allow to investigate the connection latency, state information of the mobile users and unifying technologies using IP layer. For example, the authors in [10] proposed an optimized handoff scheme using an adaptive 
retransmission timer that is proportional to the size of the messages involved in the transactions of the handoff process. This local mobility management which was placed in FMIPv6 and HMIPv6 have shown a major support to handle network layer mobility for VoIP traffic. This also allowed a minimum disruption for inter-AR movements, which are the most expected cases, and avoided triangular routing, which can harms VoIP services in mobile systems. The focus on IPv6 approach to provide solutions for mobility was also investigated in [11] where a generic framework for handoff techniques was combined with mobility management mechanisms at the IP layer in order to replace the well-performing soft handoff capabilities of UMTS/WLAN radio technologies. Although the IP handover mechanism was employed to manage the restoration of radio communication as well as proactively take actions and establish state information, the given analysis of the MIPv6 and Fast MIPv6 protocol operation focused only on handoff delays. A very similar solution using IPv6 can be also seen in [12] and [13] to support dynamic location changes of MNs and propose solutions for the impact of mobility. Analytical and simulation evaluations in [14], [15] and [16] addressed seamless mobility management by transferring the features of SIP of a separate location management function to the MIPv4. One of the advantages of such modelling is the support of a global seamless handover between homogeneous or heterogeneous networks (HetNets). However, mobility management becomes more challenging when migrating from macro-only to HetNet environments. This is due to the more challenging interference conditions, small cells appearing and disappearing more quickly as MN devices move, macro and small cells are deployed at different carrier frequencies, and so on [17]. All this literature on network-based mobility management protocols has not considered SH over IP layer.

In contrast to the aforementioned literature, this paper develops a long-term solution for the challenging spectrum handoff. Our solution aims at significantly reducing the number of spectrum handoffs in cognitive HetNets. This is performed by directly connecting the MIPv6 to the MME using a new entity that can stabilize the mobile user connections with fewer or no spectrum handoff events. The solution couples the correspondence node, target node and network management entity using IPv6 protocol as discussed in the next section

[6] L.-C. Wang, C.-W. Wang, and C.-J. Chang, "Optimal Target Channel Sequence Design for Multiple Spectrum Handoffs in Cognitive Radio Networks," Communications, IEEE Transactions on, vol. 60, no. 9, pp. 2444 - 2455, September 2012.

[7] Y. Song and J. Xie, "ProSpect: A Proactive Spectrum Handoff Framework for Cognitive Radio Ad Hoc Networks without Common Control Channel," Mobile Computing, IEEE Transactions on, vol. 11, no. 7, pp. 1127 - 1139, July 2012.

[8] S. Zheng, X. Yang, S. Chen, and C. Lou, "Target Channel Sequence Selection Scheme for ProactiveDecision Spectrum Handoff," Communications Letters, IEEE, vol. 15, no. 12, pp. 1332 - 1334, December 2011.

[9] M. NoroozOliaee, B. Hamdaoui, X. Cheng, T. Znati, and M. Guizani, "Analyzing Cognitive Network Access Efficiency Under Limited Spectrum Handoff Agility," Vehicular Technology, IEEE Transactions on, vol. 63, no. 3, pp. 1402 - 1407, March 2014.

[10]H. Fathi, S.S. Chakraborty, R. Prasad, "Optimization of Mobile IPv6-Based Handovers to Support VoIP Services in Wireless Heterogeneous Networks," Vehicular Technology, IEEE Transactions on, vol. 56, no. 1, pp. 260 - 270, Jan. 2007.

[11]L. Dimopoulou, G. Leoleis, and IS. Venieris, "Fast handover support in a WLAN environment: challenges and perspectives," Network, IEEE, vol. 19, no. 3, pp. 14 - 20, May-June 2005.

[12]M.-C. Chuang, J.-F. Lee, and M.-C. Chen, "SPAM: A Secure Password Authentication Mechanism for Seamless Handover in Proxy Mobile IPv6 Networks," IEEE Systems Journal, vol. 7, no. 1, pp. 102 - 113, March 2013. 
[13]S. Cespedes, L. Ning S. Xuemin, "VIP-WAVE: On the Feasibility of IP Communications in 802.11p Vehicular Networks," IEEE Transactions on Intelligent Transportation Systems, vol. 14, no. 1, pp. 82 97, March 2013.

[14]H. Ali-Ahmad, X. Lagrange, M. Ouzzif, and P. Bertin, "Comparative Cost Analysis on Global Mobility Management Schemes in IPv6 Mobile Networks," Vehicular Technology Conference (VTC Spring), 2013 IEEE 77th, pp. 1 - 6, 2-5 June 2013.

[15]C.-M. Huang, C.-H. Lee, and J.-R. Zheng, "A Novel SIP-Based Route Optimization for Network Mobility," Selected Areas in Communications, IEEE Journal on, vol. 24, no. 9, pp. 1682 - 1691, Sept. 2006.

[16]J.-T. Park, S.-M. Chun, J.-H. Choi, and S.-M. Lee, "Simple mobility management protocol for global seamless handover," Consumer Communications and Networking Conference (CCNC), 2012 IEEE, pp. 677 - 681, 14-17 Jan. 2012.

[17]K.I Pedersen, P.H. Michaelsen, C. Rosa, and S. Barbera, "Mobility enhancements for LTE-advanced multilayer networks with inter-site carrier aggregation," Communications Magazine, IEEE, vol. 51, no. 5, pp. 64 - 71, May 2013.

Comments 2: In section 3.C, the description of the proposed new Handoff Scheme is abstract. Detailed description is needed.

Response: Thanks for your comments. This contents of the whole section 3 and specially 3.C has been improved and also the figures 4, 5, and 6 where improved to allow the challenge and soution used to create Algorithms $1 \& 2$.

Comments 3: In section 5, the description of two algorithms are not clear. And performance of the algorithms should be analyzed.

Response: Thanks for your comments. We improved the description of the algorithms and how the different operations are connected to the channel assessment model and the enforcement/creasing of spectrum handoff actions. These algorithms are the pseudo-code highlights of the channel functions that were incorporated within the OPNET simulator to allow large network size evaluations. The details of the network nodes and parameters values are given in Table II. Then, Table III describes the way we setup the trajectories for cognitive mobile nodes moving between different macro/femto domains. Finally, the Algorithms $1 \& 2$ performances were presented as <no $\mathrm{SH}>$ and <with $\mathrm{SH}>$ in Figures 10, 11, 12 and 13. 
Comments 4: In section 6, the subsections $A$ and $B$ have the same title. A related algorithm in other literature (such as ref. [21]) also should be used to compare with the proposed scheme.

Response: Thanks for your comments. We are really sorry to notice that we have repeated the same subtitles in Section 6 twice. Subsections A and B titles are changed now as "A. Network Setup" and "B. System Level Simulation".

The algorithm from ref. [21]: <O. Jo and D.-H. Cho, "Seamless spectrum handover considering differential path-loss in cognitive radio systems," Communications Letters, IEEE, vol. 13, no. 3, pp. 190 - 192, March 2009> proposes an initial solution for intercell spectrum handoff action. We mentioned this algorithm to show the significance of our model as we expanded the solution of Ref: [21] from intercell to multi-subcell domains (femtocells) that are being contained within one macrocell. For instance, Fig. 4 shown Ref: [21] solution while our solutions are presented in Figures $5 \& 6$. So, we are not able to compare any results with Ref: [21] as it has a very limited scope of application to only one base station while our proposed solution throughout this paper investigates large-sized network of many femtocells and mobile node station as shown in Table II. Therefore and to avoid any confusion, we changed Section III-B to show the novelty of our model and the scope of our application compared to Ref: [21] as follows:

\section{Model Formulation}

We investigate the spectrum handoff problem that is likely to occur for a MN travelling between two cognitive base stations. Our intension is to develop the necessary control signal scheme for channel allocation for HetNet. From the literature, the authors in [21] proposed an intercell spectrum handoff scheme as shown in Fig. 3. In this scheme, the mobile user senses the spectrum periodically to detect the presence of any primary user. The sensing results are exchanged with $\mathrm{CN}$ which may decides to allocate another channel to the MN. In this case, the availability of the new channel will be negotiated with neighbor nodes to prevent interference. If it was decided to adapt to a new channel, there will be a distribution in the services during the time of frequency adaptation. This disrupted mobile user needs to carry out additional intercell handoff to maintain a connection. In the worst case, the mobile users must carry out a new network entry procedure due to the connection loss. Clearly, this was developed to solve the problem when a primary user reclaims its channel and the cognitive $\mathrm{MN}$ has to look for another channel to maintain cognitive communications. We think that this scheme is an intial step to generate the signal control scheme for the spectrum handoff and we expand this work by incorporating a channel reservation mechanism that can reduce not only the probability of service interruption, but also the total number of potential handoffs. 


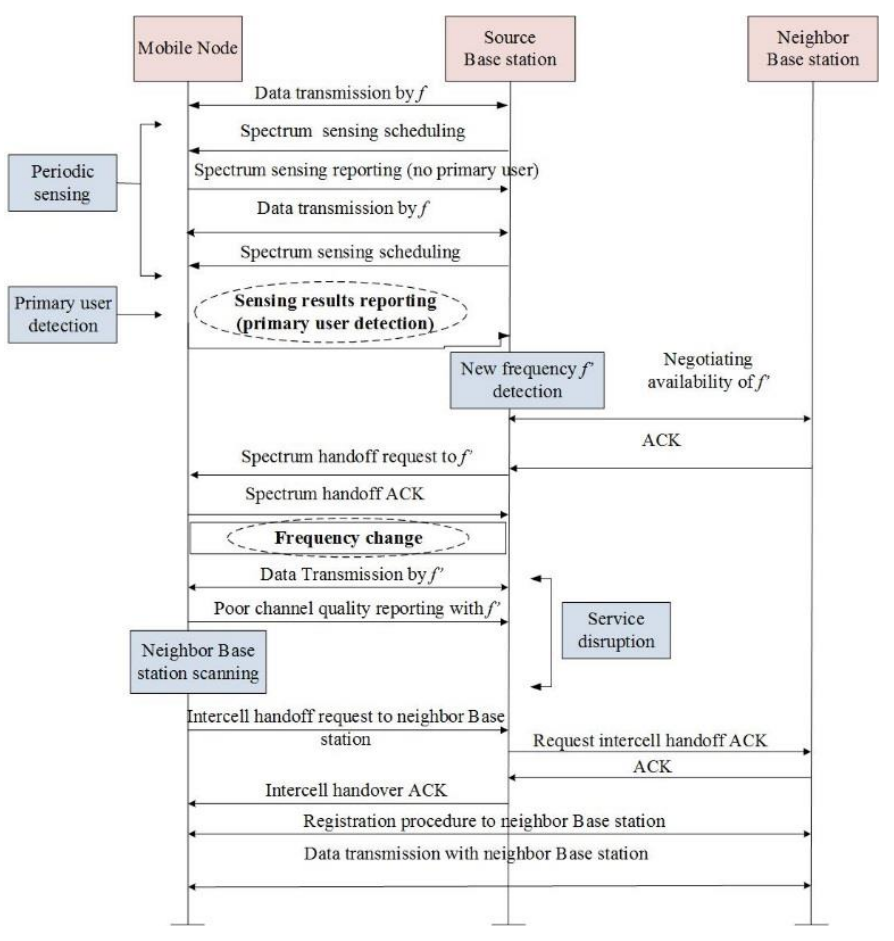

Fig. 3. Operation of intercell spectrum handoff [21]. 


\section{Reviewer 3:}

Comments 1: In section 1, more detailed description on motivation is needed, although the contribution is clear.

Response: Thanks for your comments. We are sorry that we did not present enough information about our motivation for this contribution. In this revised version, we provide more description about the main idea and contributions in detail as follows.

\section{INTRODUCTION}

Cognitive radio users may encounter multiple connections interruptions when channels becomes unavailable due to the primary users transmissions or normal handoff operations. These interruptions triggers in a series of Spectrum Handoffs ( $S H s$ ) to restore cognitive communications whenever an interruption event occurs [1], [2]. Clearly, spectrum handoffs will increase the time required for reconnecting the newly arrival mobile users in any cognitive transmission domain while re-allocating channels. This new factor of time delay increases the time required for data delivery especially in cognitive networks that employ small cells such as femtocells. The main concern for such model of networks is that interruption may occur for unpredicted time intervals leading to significant signaling overhead and degradation in the whole system performance.

It reasonable to incorporate any new mobility functionality with the IP layer in order to solve the problem of spectrum handover. This is due to the fact that the IP layer is generic enough to serve all underlying technologies [3]. Also, it is widely accepted that there is a strong need to migrate the technology-specific core infrastructures toward all-IP networks [4]. The mobile IP working group within the internet engineering task force (IETF) proposed a packet-based mobility management protocol namely mobile internet protocol (MIP) in order to support global mobility in IP networks. This was upgraded later on to MIPv6 in response to the emergence of IPv6. In MIP, each mobile node (MN) is identified using two different IP addresses: a permanent home address (HoA) and a temporary care of address (CoA). The CoA is provided to the $\mathrm{MN}$ as it roams in a foreign network other than its home network. The CoA is given to the MN by the visiting subnet after issuing a router solicitation (RS) message to its foreign agent (FA) [5].

Even with the flexibility in performing IP handover, developing a solution for spectrum handoff in cognitive radio networks is still a complex challenge as it needs to answer those two questions: how to transfer the updated state information of the mobile users' used channel from the MIP to the Mobility Management Entity (MME)? what entities should be involved to allocate a certain channel for a mobile user that is moving between two cognitive access points? Considering the motivation to avoid the impacts of spectrum handover and the complexity of the above challenges, we propose a new scheme that can allocates the same channel to a cognitive mobile user moving between various access points as long as this channel is vacant in these domains. This goals that we intend to achieve here are:

- Reducing the number of unnecessary spectrum handoffs and improving the cognitive communications stability.

- Minimizing the time latency incurred during the data delivery.

- Improving spectrum coordination and avoiding channels scattering due to unnecessary spectrum handoff. 
Therefore, the main contribution of this paper is to propose a solution to the spectrum handoff problem in cognitive radio networks overlaid with femtocells. We propose a new channel assigning agent (CAA) entity at the IP protocol layers. The CAA retain the same channel in use by a mobile unit when it movies to a new macro/femto transmission domain whenever the requested channel is available. The CAA is coupled with the MME to allow centralized management of the channel allocation during handoff in large cognitive networks. This can minimize or eliminate the time consumed during spectrum handoff in a cognitive radio network that employs femtocells. Our simulation results demonstrate that the proposed scheme reduces the total handoff latency, end-to-end data time delay, number of handoffs and improves the overall system throughput. The contents of this paper are arranged into stages in order to develop the CAA system model as shown in Fig. 1.

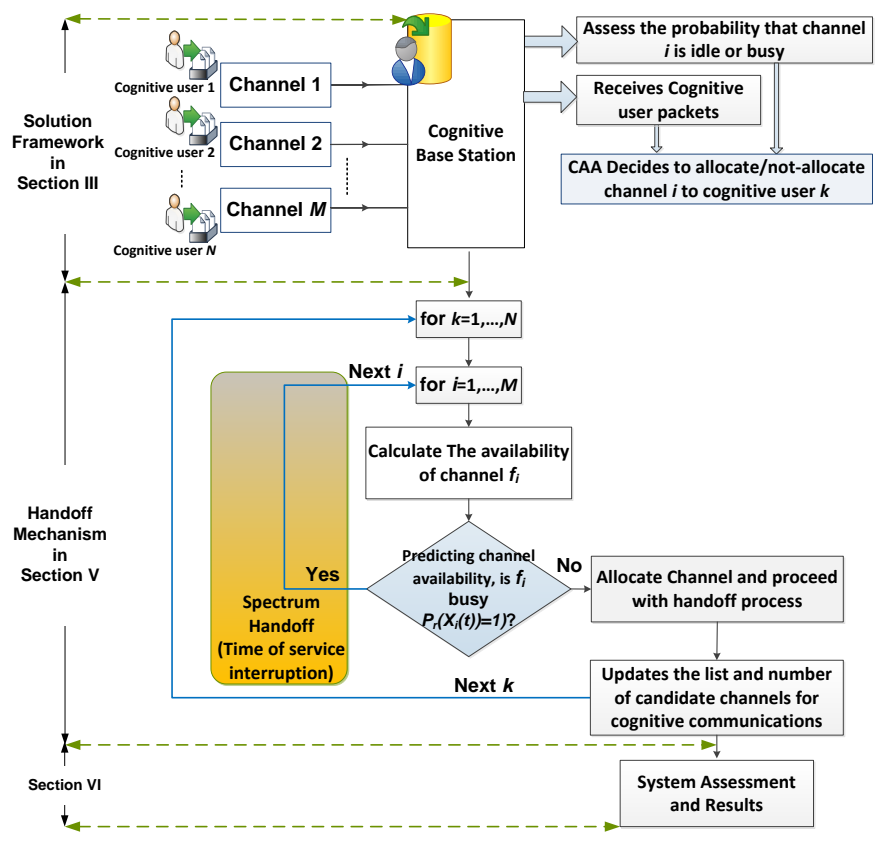

Fig. 1. Overview of contents in this paper.

The remainder of this paper is organized as follows. Section II discusses related studies. Section III overviews the IPv6 system model and the flow diagrams of the proposed scheme. Section IV describes the protocol of spectrum mobility and handoff in cognitive networks. The algorithms of spectrum handoff management are given in Section V. Simulation evaluations and performance analyses are presented in Section VI. Finally, our conclusions are presented in Section VII.

[1] C.-W. Wang and L.-C. Wang, "Analysis of Reactive Spectrum Handoff in Cognitive Radio Networks," Selected Areas in Communications, IEEE Journal on, vol. 30, no. 10, pp. 2016 - 2028, November 2012.

[2] Y.-S. Chen and J.-S. Hong, "A Relay-Assisted Protocol for Spectrum Mobility and Handover in Cognitive LTE Networks," Systems Journal, IEEE, vol. 7, no. 1, pp. 77 - 91, March 2013.

[3] Z. Yan and J.-H. Lee, "State-Aware Pointer Forwarding Scheme With Fast Handover Support in a PMIPv6 Domain," Systems Journal, IEEE, vol. 7, no. 1, pp. 92 - 101, March 2013. 
[4] C. Makaya and S. Pierre, "An Architecture for Seamless Mobility Support in IP-Based Next-Generation Wireless Networks," Vehicular Technology, IEEE Transactions on, vol. 57, no. 2, pp. 1209 - 1225, March 2008.

[5] T. Taleb, and K. Letaief, "A cooperative diversity based handoff management scheme," Wireless Communications, IEEE Transactions on, vol. 9, no. 4, pp. 1462 - 1471, April 2010.

Comments 2: In section 2, some recent related work did not reviewed.

Response: Thanks for your comments. In the revised paper, we added the most recent related work that has been published in highly ranked IEEE journals. Considering the limited space in the original manuscript, we are trying to survey only the papers that can provide the readers with enough background about the paper topic and how to integrate different ideas into one major system that can solve the problem of spectrum handoff. The new section is given below.

\section{RELATED STUDIES}

There are no literature that solve the problem of spectrum handoff using MIP according to the author's knowledge. Keeping in mind that there are few studies addressing solutions to the spectrum handoff issue, we will start by showing the most prominent solutions for spectrum handoff and then we show how MIP is used to solve the handoff latency. Our goal is to establish the necessary background for a solution that incorporates both SH and MIP as shown in the following sections. Some of the most related studies to our work are described below

An optimization problem was formulated in [6] for a proactive determination of target channel selection with the objective of minimizing the cumulative delay per connection. This allows a newly arriving secondary user to avoid multiple spectrum handoffs due to the high-priority primary users and the traffic statistics of both the primary and the secondary users. This process takes into account the time required for channel switching and the transmission delay time resulting from accessing unsuitable channels. In contrast to a proactive assignment before accessing the channel, channel-switching $(\mathrm{CW})$ policies and a proactive spectrum handoff protocol in [7] were proposed to let cognitive users vacate a channel before the primary user access it to avoid interference. This means that that cognitive user is using the channel and it acts before the primary user returns to occupy the space. Once the cognitive user driven into conduct a spectrum handoff, a distributed channel selection scheme is activated to avoid collisions among cognitive users in a multiuser spectrum handoff scenario. A further application of the optimal target channel sequence selection in proactive-decision spectrum handoff is given in [8] with a Poisson arriving of primary users. The theoretical analysis has shown a minimum probability of spectrum handoffs failure using the proposed scheme. The authors in [9] considered the channel handoff agility limitations for a cognitive radio user with a dynamic multichannel-access capability. The channel handoff agility was modeled as a continuous-time Markov process in order to analytically derive the forced access termination and blocking probabilities of cognitive users. Although the paper assumptions accepts that spectrum handoffs can only be performed to vacant channels that are immediate neighbors of the cognitive users' current channels but does not show how this scenario can reduce the numbers of spectrum handoffs or proactively prevent them. Clearly, the spectrum handoff studies investigates methods that can host the arrival of a cognitive user in the spectrum or the ways to response to subsequent changes in the spectrum availability without being able to prevent 
spectrum handoff occurrence.

The handling of handoff operations with the MIP have a very different prospective from the spectrum handoff prospective that we mentioned earlier due to the operation management. The MIP features allow to investigate the connection latency, state information of the mobile users and unifying technologies using IP layer. For example, the authors in [10] proposed an optimized handoff scheme using an adaptive retransmission timer that is proportional to the size of the messages involved in the transactions of the handoff process. This local mobility management which was placed in FMIPv6 and HMIPv6 have shown a major support to handle network layer mobility for VoIP traffic. This also allowed a minimum disruption for inter-AR movements, which are the most expected cases, and avoided triangular routing, which can harms VoIP services in mobile systems. The focus on IPv6 approach to provide solutions for mobility was also investigated in [11] where a generic framework for handoff techniques was combined with mobility management mechanisms at the IP layer in order to replace the well-performing soft handoff capabilities of UMTS/WLAN radio technologies. Although the IP handover mechanism was employed to manage the restoration of radio communication as well as proactively take actions and establish state information, the given analysis of the MIPv6 and Fast MIPv6 protocol operation focused only on handoff delays. A very similar solution using IPv6 can be also seen in [12] and [13] to support dynamic location changes of MNs and propose solutions for the impact of mobility. Analytical and simulation evaluations in [14], [15] and [16] addressed seamless mobility management by transferring the features of SIP of a separate location management function to the MIPv4. One of the advantages of such modelling is the support of a global seamless handover between homogeneous or heterogeneous networks (HetNets). However, mobility management becomes more challenging when migrating from macro-only to HetNet environments. This is due to the more challenging interference conditions, small cells appearing and disappearing more quickly as MN devices move, macro and small cells are deployed at different carrier frequencies, and so on [17]. All this literature on network-based mobility management protocols has not considered SH over IP layer.

In contrast to the aforementioned literature, this paper develops a long-term solution for the challenging spectrum handoff. Our solution aims at significantly reducing the number of spectrum handoffs in cognitive HetNets. This is performed by directly connecting the MIPv6 to the MME using a new entity that can stabilize the mobile user connections with fewer or no spectrum handoff events. The solution couples the correspondence node, target node and network management entity using IPv6 protocol as discussed in the next section

[6] L.-C. Wang, C.-W. Wang, and C.-J. Chang, "Optimal Target Channel Sequence Design for Multiple Spectrum Handoffs in Cognitive Radio Networks," Communications, IEEE Transactions on, vol. 60, no. 9, pp. 2444 - 2455, September 2012.

[7] Y. Song and J. Xie, "ProSpect: A Proactive Spectrum Handoff Framework for Cognitive Radio Ad Hoc Networks without Common Control Channel," Mobile Computing, IEEE Transactions on, vol. 11, no. 7, pp. 1127 - 1139, July 2012.

[8] S. Zheng, X. Yang, S. Chen, and C. Lou, "Target Channel Sequence Selection Scheme for ProactiveDecision Spectrum Handoff," Communications Letters, IEEE, vol. 15, no. 12, pp. 1332 - 1334, December 2011.

[9] M. NoroozOliaee, B. Hamdaoui, X. Cheng, T. Znati, and M. Guizani, "Analyzing Cognitive Network Access Efficiency Under Limited Spectrum Handoff Agility," Vehicular Technology, IEEE Transactions on, vol. 63, no. 3, pp. 1402 - 1407, March 2014.

[10]H. Fathi, S.S. Chakraborty, R. Prasad, "Optimization of Mobile IPv6-Based Handovers to Support VoIP Services in Wireless Heterogeneous Networks," Vehicular Technology, IEEE Transactions on, vol. 56, no. 1, pp. 260 - 270, Jan. 2007.

[11]L. Dimopoulou, G. Leoleis, and IS. Venieris, "Fast handover support in a WLAN environment: challenges and perspectives," Network, IEEE, vol. 19, no. 3, pp. 14 - 20, May-June 2005. 
[12]M.-C. Chuang, J.-F. Lee, and M.-C. Chen, "SPAM: A Secure Password Authentication Mechanism for Seamless Handover in Proxy Mobile IPv6 Networks," IEEE Systems Journal, vol. 7, no. 1, pp. 102 - 113, March 2013.

[13]S. Cespedes, L. Ning S. Xuemin, "VIP-WAVE: On the Feasibility of IP Communications in 802.11p Vehicular Networks," IEEE Transactions on Intelligent Transportation Systems, vol. 14, no. 1, pp. 82 97, March 2013.

[14]H. Ali-Ahmad, X. Lagrange, M. Ouzzif, and P. Bertin, "Comparative Cost Analysis on Global Mobility Management Schemes in IPv6 Mobile Networks," Vehicular Technology Conference (VTC Spring), 2013 IEEE 77th, pp. 1 - 6, 2-5 June 2013.

[15]C.-M. Huang, C.-H. Lee, and J.-R. Zheng, "A Novel SIP-Based Route Optimization for Network Mobility," Selected Areas in Communications, IEEE Journal on, vol. 24, no. 9, pp. 1682 - 1691, Sept. 2006.

[16]J.-T. Park, S.-M. Chun, J.-H. Choi, and S.-M. Lee, "Simple mobility management protocol for global seamless handover," Consumer Communications and Networking Conference (CCNC), 2012 IEEE, pp. 677 - 681, 14-17 Jan. 2012.

[17]K.I Pedersen, P.H. Michaelsen, C. Rosa, and S. Barbera, "Mobility enhancements for LTE-advanced multilayer networks with inter-site carrier aggregation," Communications Magazine, IEEE, vol. 51, no. 5, pp. 64 - 71, May 2013.

Comments 3: In section 3, the framework is simply based on the work in [20] and [21]. And the difference between the new scheme in 3.C and [21] is not so clear.

Response: Thanks for your comments. In the revised paper, we added the most recent related work that has been published in highly ranked IEEE journals. Considering the limited space in the original manuscript, we are trying to survey only the papers that can provide the readers with enough background about the paper topic and how to integrate different ideas into one major system that can solve the problem of spectrum handoff. The new section is given below.

Comments 4: In section 4, the probability threshold in (4) should be explained in detail. And how to optimize the probability threshold.

Response: Thanks for your comments. We are sorry that we did not provide enough information in the previous version on how to use the threshold to decide upon the channel availability for cognitive transmission during spectrum handoff approval. In this revised version provide a more 
specific details about the policy to consider a channel available with details on the threshold for packet time and probability to consider a channel as idle with mathematical assessment. This included in Section IV as following:

To estimate the probability that a channel is idle, it is necessary to identify the time intervals of busy and idle states of random transmission durations. Considering Fig. 7 and using a Bayesian learning algorithm [22], the probability that channel $i$ is idle can be given as follows:

$$
P_{f_{i}}^{i d l e}(t)=\frac{X_{i}(t)+1}{X_{b}(t)+X_{i}(t)+2}
$$

where $X_{b}(t)$ and $X_{i}(t)$ are the numbers of slot times that channel $i$ is busy or idle in a future time interval $t$.

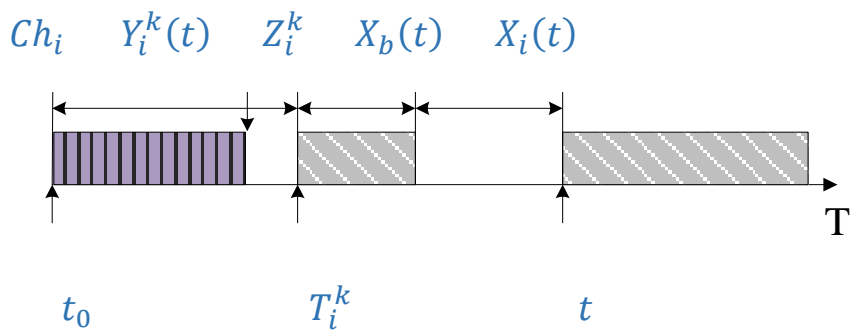

Fig. 7. Primary user traffic activity on channel $f_{i}$,

TABLE I

MYTHOLOGY

$Y_{i}^{k}(t)$ Number of successfully transmitted packets of a number of cognitive

user $k$ over channel $i$

$Y_{f}^{k}(t)$ Number of failed transmitted packets of a number of cognitive user $k$ over channel $i$

$T_{i}^{k} \quad$ Packets arrival time of a number of cognitive user $k$ over channel $i$

$X_{b}(t)$ Number of slot times that channel $i$ is busy in a future time interval $t$

$X_{i}(t)$ Number of slot times that channel $i$ is idle in a future time interval $t$

$k^{\text {th }} \quad$ Number of cognitive users

$i^{\text {th }} \quad$ Number of channels available to cognitive users

$\underline{Z_{i}^{k} \quad \text { Packet length of a cognitive user } k \text { over channel } i}$

Therefore, the criterion for channel $i^{\text {th }}$ to become a candidate channel at time interval $t$ is given by:

$$
\left\{P_{f_{i}}^{\text {success }}(t)=\frac{Y_{i}^{k}(t)+1}{Y_{i}^{k}(t)+Y_{f}^{k}(t)+2} \text { and } T_{i}^{k} \leq t\right.
$$




$$
Z_{i}^{k} \leq \tau_{z}
$$

where $Y_{i}^{k}(t)$ and $Y_{f}^{k}(t)$ are the number of successful and failed cognitive radio slot transmissions over channel $i, Z_{i}^{k}$ represents the packet length of the a number $k^{t h}$ of cognitive users on channel $i$ and $\tau_{z}$ is the maximum packet length that can be conveyed over a link ( $\tau_{z}=10 \mathrm{~ms}$ for SIP).

Therefore, the probability of successful cognitive transmission over certain link using (4) can be shown in Fig. 8.

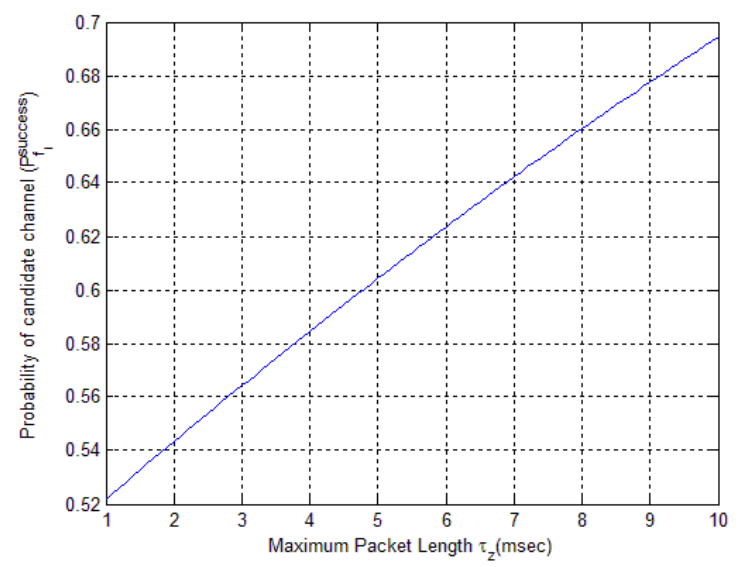

Fig. 8. Probability of successful candidate channel for cognitive transmission over interval time $t$

In this case, there is no need to perform a spectrum handoff operation and the cognitive user is being facilitated easily in coexistence with the primary user. The condition in (4) means that, in order to support at least one cognitive user frame, the probability that the duration of the idleness of the $i^{\text {th }}$ channel has to be longer than a frame size must be higher than or equal to $\tau_{z}$.

This means that a spectrum handoff action will be necessary whenever:

$$
\begin{gathered}
\left(P_{f_{i}}^{\text {success }}(t)=0\right) \text { if } T_{i}^{k}<t \text { and } T_{i}^{k}+\mathrm{Z}_{i}^{k} \geq \mathrm{t}, \quad k \geq 1 \\
\left(P_{f_{i}}^{\text {success }}(t)=0\right) \text { if } T_{i}^{k}+\mathrm{Z}_{i}^{k}<t \text { and } T_{i}^{k+1} \geq \mathrm{t}, k \geq 1, \\
T_{i}^{k+1} \geq \mathrm{t}, \quad k=0,
\end{gathered}
$$

In this case it is necessary to perform spectrum handoff provided by scanning other channels in the available band to identify a new candidate channel in order to resume transmission. It should be also notice that a cognitive user should switch to a new channel if:

$$
Z_{i}^{k} \geq \tau_{z}
$$


The above polices are used to develop the probe for channel assignment in spectrum handoff model. This performed in the CAA protocol given in the following section.

Comments 5: In section 5, the performance of the protocol model should be analyzed mathematically.

Response: Thanks for your comments. The proposed protocol is based on functions modification in the IPv6 for mobility management. Therefore, the system design incorporates a modifications to the follow of control signals during handoff request in cognitive radio networks, decision making at the MME unit, and channel assessment at PHY layer to decide upon channel availability. Therefore, it is almost impossible to create a one mathematical model that can incorporate all these functions and merit of success. This is the main reason that we address this paper to the IEEE systems Journal in order to show that a complex integration of different systems can provide a novel solution to a very challenging problem such as Spectrum Handoff. However, we thank you very much for this suggestion and we will keep trying to find a new aspect for an optimisation model that can solve the spectrum handoff problem for one channel profile in our future work. We wish that Fig. 8 which is based on mathematical calculations can provide a basis for our future work in this field.

Comments 6: In section 6, a more related and recent scheme in other literature should be used to compare with the proposed schemes to prove the effectiveness of the proposed scheme.

Response: Thanks for your comments. We are really sorry for not presenting enough information in the paper about this case. In this revised version we make it clear to the readers that <with "SH" $>$ is meant to describe our proposed cognitive radio networks that can avoid spectrum handoff actions during handoff operations between different wireless domains. Such amendment to handoffs can happen only with the new proposed CAA entity as clarified in the paper. While $<$ without "SH" $>$ describes the traditional cognitive radio networks where handoff operations 
incorporates spectrum handoff actions when a mobile node performs cell/intercell handoffs. As developed our algorithms using OPNET software, we were able to create the traditional cognitive radio systems with $\mathrm{SH}$ and then we developed the new nodes with the CAA to enable the channel reservation model that can maintain the same channel and avoid SH. Both models were simulated, at one time to show the advantages of the new development under the same channel availability profiles and traffic loads. This was described in Section VI in details. 